\title{
Global $\mu$-Stability of Impulsive Complex-Valued Neural Networks with Leakage Delay and Mixed Delays
}

\author{
Xiaofeng Chen,, Qiankun Song, ${ }^{1}$ Yurong Liu, ${ }^{2,3}$ and Zhenjiang Zhao ${ }^{4}$ \\ ${ }^{1}$ Department of Mathematics, Chongqing Jiaotong University, Chongqing 400074, China \\ ${ }^{2}$ Department of Mathematics, Yangzhou University, Yangzhou 225002, China \\ ${ }^{3}$ Faculty of Engineering, King Abdulaziz University, Jeddah 21589, Saudi Arabia \\ ${ }^{4}$ Department of Mathematics, Huzhou Teachers College, Huzhou 313000, China
}

Correspondence should be addressed to Qiankun Song; qiankunsong@163.com

Received 10 April 2014; Accepted 5 May 2014; Published 27 May 2014

Academic Editor: Zidong Wang

Copyright (C) 2014 Xiaofeng Chen et al. This is an open access article distributed under the Creative Commons Attribution License, which permits unrestricted use, distribution, and reproduction in any medium, provided the original work is properly cited.

\begin{abstract}
The impulsive complex-valued neural networks with three kinds of time delays including leakage delay, discrete delay, and distributed delay are considered. Based on the homeomorphism mapping principle of complex domain, a sufficient condition for the existence and uniqueness of the equilibrium point of the addressed complex-valued neural networks is proposed in terms of linear matrix inequality (LMI). By constructing appropriate Lyapunov-Krasovskii functionals, and employing the free weighting matrix method, several delay-dependent criteria for checking the global $\mu$-stability of the complex-valued neural networks are established in LMIs. As direct applications of these results, several criteria on the exponential stability, power-stability, and log-stability are obtained. Two examples with simulations are provided to demonstrate the effectiveness of the proposed criteria.
\end{abstract}

\section{Introduction}

The nonlinear systems are ubiquitous in the real world [15]. As one of the most important nonlinear systems, the real-valued neural networks have been extensively studied and developed due to their extensive applications in pattern recognition, associative memory, signal processing, image processing, combinatorial optimization, and other areas [6]. In implementation of neural networks, however, time delays are unavoidably encountered [7]. It has been found that the existence of time delays may lead to instability and oscillation in a neural network [8]. Therefore, dynamics analysis of neural networks with time delays has received much attention. In [6-10], the exponential stability and asymptotic stability of delayed neural networks were investigated; some sufficient conditions for checking stability were given. In $[11,12]$, authors investigated the synchronization of chaotic neural networks with delay and obtained several criteria for checking the synchronization. In [13], the passivity of uncertain neural networks with both leakage delay and timevarying delay was considered. In [14], authors investigated the state estimation for neural networks with leakage delay and time-varying delays.

However, besides delay effect, impulsive effects are also likely to exist in neural networks [15]. For instance, in implementation of electronic networks, the state of the networks is subject to instantaneous perturbations and experiences abrupt change at certain instants, which may be caused by switching phenomenon, frequency change. or other sudden noise; that is, it exhibits impulsive effects [16-18]. Therefore, it is necessary to consider both impulsive effect and delay effect on dynamical behaviors of neural networks. Some results on impulsive effect have been gained for delayed neural networks; for example, see $[15,16,19]$ and the references therein.

Recently, the power-rate global stability of the equilibrium is proposed in [20]. Moreover, in [21], the authors proposed a new concept of global $\mu$-stability to unify the exponential stability, power-rate stability, and log-stability of neural networks. In [22], the authors investigated the global robust $\mu$-stability in the mean square for a class of stochastic neural networks. In [23], the delayed neural systems with 
impulsion were considered, and the $\mu$-stability criteria were derived by using Lyapunov-Krasovskii functional method. In [24], the multiple $\mu$-stability of delayed neural networks was investigated, and several criteria for the coexistence of equilibrium points and their local $\mu$-stability were derived.

As an extension of real-valued neural networks, complexvalued neural networks with complex-valued state, output, connection weight, and activation function become strongly desired because of their practical applications in physical systems dealing with electromagnetic, light, ultrasonic, and quantum waves $[25,26]$. It has been shown that such applications strongly depend on the stability of CVNNs [27]. Therefore, stability analysis of CVNNs has received much attention and various stability conditions have been obtained [28-33]. In [28], authors considered a discrete-time CVNNs and obtained several sufficient conditions for checking global exponential stability of a unique equilibrium. In $[29,30]$, the discrete-time CVNNs with linear threshold neurons were investigated, and some conditions for the boundedness, global attractivity, and complete stability as well as global exponential stability of the considered neural networks were also derived. In [31], the continuous-time CVNNs with delays were considered, and the boundedness, complete stability, and exponential stability were investigated. In [32, 33], the global stability was investigated for CVNNs on time scales, which is useful to unify the continuous-time and discretetime CVNNs under the same framework. In [34], the authors investigated the $\mu$-stability of delayed CVNNs and obtained several sufficient conditions to ensure the global $\mu$-stability. To the best of our knowledge, there are no results on $\mu$ stability of impulsive CVNNs with the three kinds of time delays including leakage delay, discrete delay, and distributed delay in the literature, and it remains as an open topic for further investigation.

Motivated by the above discussions, in this paper, we will deal with the problem of $\mu$-stability for CVNNs with leakage delay, discrete delay, and distributed delay under impulsive perturbations. Based on the homeomorphism mapping principle of complex domain, a LMI condition for the existence and uniqueness of the equilibrium point of the addressed CVNNs is proposed. Several delay-dependent criteria for checking the global $\mu$-stability of the CVNNs are obtained by constructing appropriate Lyapunov-Krasovskii functionals and employing the free weighting matrix method. The obtained results can also be applied to several special cases and we can get the exponential stability, power-stability, and log-stability of the CVNNs, correspondingly. Finally, two illustrative examples are provided to show the effectiveness of the proposed criteria.

Notations. The notations are quite standard. Throughout this paper, let $\mathbb{Z}^{+}$denote the set of positive integers. Let $i$ denote the imaginary unit; that is, $i=\sqrt{-1} \cdot \mathbb{C}^{n}, \mathbb{R}^{m \times n}$, and $\mathbb{C}^{m \times n}$ denote, respectively, the set of $n$-dimensional complex vectors, $m \times n$ real matrices and complex matrices. The subscripts $T$ and $*$ denote matrix transposition and matrix conjugate transpose, respectively. For complex vector $z \in \mathbb{C}^{n}$, let $|z|=\left(\left|z_{1}\right|,\left|z_{2}\right|, \ldots,\left|z_{n}\right|\right)^{T}$ be the module of the vector $z$, and let $\|z\|=\sqrt{\sum_{k=1}^{n}\left|z_{k}\right|^{2}}$ be the norm of the vector $z$. $I$ denotes the identity matrix with appropriate dimensions. The notation $X \geq Y$ (resp., $X>Y$ ) means that $X-Y$ is positive semidefinite (resp., positive definite). $\lambda_{\max }(P)$ and $\lambda_{\text {min }}(P)$ are defined as the largest and the smallest eigenvalue of positive definite matrix $P$, respectively. For any $t \geq 0, z_{t}$ is defined by $z_{t}=z(t+s), z_{t^{-}}=z\left(t^{-}+s\right), s \in[-\delta, 0]$. In addition, the notation $\star$ always denotes the conjugate transpose of block in a Hermitian matrix.

\section{Problems Formulation and Preliminaries}

Consider the following complex-valued neural networks with leakage delay and mixed delays under impulsive perturbations by a nonlinear differential equation of the form

$$
\begin{aligned}
\dot{z}(t)= & -D z(t-\delta)+A f(z(t))+B f\left(z\left(t-\tau_{1}\right)\right) \\
& +C \int_{t-\tau_{2}}^{t} f(z(s)) d s+h, \quad t \neq t_{k}, \quad t>0, \\
\Delta z\left(t_{k}\right)= & z\left(t_{k}\right)-z\left(t_{k}^{-}\right)=J_{k}\left(z\left(t_{k}^{-}\right), z_{t_{k}^{-}}\right), \quad k \in \mathbb{Z}^{+},
\end{aligned}
$$

where the impulse times $t_{k}$ satisfy $0=t_{0}<t_{1}<\cdots<t_{k}<$ $\cdots$ and $\lim _{k \rightarrow \infty} t_{k}=+\infty ; z(t)=\left(z_{1}(t), z_{2}(t), \ldots, z_{n}(t)\right)^{T} \epsilon$ $\mathbb{C}^{n}$ is the state vector of the neural networks; $D=$ $\operatorname{diag}\left\{d_{1}, d_{2}, \ldots, d_{n}\right\} \in \mathbb{R}^{n \times n}$ is the self-feedback connection weight matrix with $d_{j}>0(j=1,2, \ldots, n) ; A, B$, and $C \in \mathbb{C}^{n \times n}$ are, respectively, the connection weight matrix, the discretely delayed connection weight matrix, and distributively delayed connection weight matrix; $f(z(t))$ $=\left(f_{1}\left(z_{1}(t)\right), f_{2}\left(z_{2}(t)\right), \ldots, f_{n}\left(z_{n}(t)\right)\right)^{T} \in \mathbb{C}^{n}$ represents the neuron activation function; $h=\left(h_{1}, h_{2}, \ldots, h_{n}\right)^{T} \in \mathbb{C}^{n}$ is the external input vector; $\delta \geq 0, \tau_{1} \geq 0$, and $\tau_{2} \geq 0$ are the leakage time delay, the discrete time delay, and the distributed time delay, respectively; $J_{k}$ is the impulsive function.

In the analysis of complex-valued neural networks, it is usually assumed that the activation functions are differentiable [31]. However, in this paper, we adopt the following assumption on the activation functions in which the differentiability is not be required:

(H1) the neuron activation functions $f_{j}$ are continuous and satisfy

$$
\left\|f_{j}\left(z_{1}\right)-f_{j}\left(z_{2}\right)\right\| \leq \gamma_{j}\left\|z_{1}-z_{2}\right\|,
$$

for any $z_{1}, z_{2} \in \mathbb{C}, j=1,2, \ldots, n$, where $\gamma_{j}$ is a constant. Moreover, we define $\Gamma=\operatorname{diag}\left\{\gamma_{1}^{2}, \gamma_{2}^{2}, \ldots, \gamma_{n}^{2}\right\}$.

Remark 1. Note that the assumptions on activation functions are weaker than those generally used in the literature. Namely, the boundedness and differentiability of the activation functions $f_{j}$ are not required in this paper.

Next we introduce some definitions and lemmas to be used in the stability analysis. 
Definition 2. Let $\widehat{z}$ be an equilibrium point of system (1). Suppose that $z(t)$ is an arbitrary solution of system $(1) ; \mu(t)$ is a positive continuous function and satisfies $\mu(t) \rightarrow \infty$ as $t \rightarrow \infty$. If there is a positive constant $M$ such that

$$
\|z(t)-\widehat{z}\| \leq \frac{M}{\mu(t)}, \quad t \geq 0,
$$

then the equilibrium point $\widehat{z}$ is said to be $\mu$-stable.

In particular, if taking $\mu(t)$ in Definition 2 to exponential function, power function, and logarithmic function, we can get the definitions of exponential stability, power-stability, and log-stability, correspondingly.

Definition 3. Let $\widehat{z}$ be an equilibrium point of system (1). Suppose that $z(t)$ is an arbitrary solution of system (1). If there are two positive constants $\mathcal{\varepsilon}$ and $M$ such that

$$
\|z(t)-\widehat{z}\| \leq \frac{M}{e^{\varepsilon t}}, \quad t \geq 0
$$

then the equilibrium point $\widehat{z}$ is said to be exponentially stable.

Definition 4. Let $\widehat{z}$ be an equilibrium point of system (1). Suppose that $z(t)$ is an arbitrary solution of system (1). If there are two positive constants $\varepsilon$ and $M$ such that

$$
\|z(t)-\widehat{z}\| \leq \frac{M}{t^{\varepsilon}}, \quad t \geq 0
$$

then the equilibrium point $\widehat{z}$ is said to be power-stable.

Definition 5. Let $\widehat{z}$ be an equilibrium point of system (1). Suppose that $z(t)$ is an arbitrary solution of system (1). If there are two positive constants $\varepsilon$ and $M$ such that

$$
\|z(t)-\widehat{z}\| \leq \frac{M}{\ln (\varepsilon t+1)}, \quad t>0,
$$

then the equilibrium point $\widehat{z}$ is said to be log-stable.

Lemma 6. If $H(z): \mathbb{C}^{n} \rightarrow \mathbb{C}^{n}$ is a continuous map and satisfies the following conditions:

(i) $H(z)$ is injective on $\mathbb{C}^{n}$,

(ii) $\lim _{\|z\| \rightarrow \infty}\|H(z)\|=\infty$,

then $H(z)$ is a homeomorphism of $\mathbb{C}^{n}$ onto itself.

Proof. Let $z=x+i y$ and $\alpha=\left(x^{T}, y^{T}\right)^{T}$, where $x, y \in \mathbb{R}^{n}$. Define a homeomorphism $\mathscr{I}: \mathbb{C}^{n} \rightarrow \mathbb{R}^{2 n}$ by

$$
\mathscr{I}(z)=\alpha
$$

Obviously, $\mathscr{I}$ is invertible. Let $\mathscr{L}=\mathscr{I} \circ H \circ \mathscr{I}^{-1}$. Then $\mathscr{L}$ is injective on $\mathbb{R}^{2 n}$, since $H$ and $\mathscr{I}$ are injective. In addition, $\|\mathscr{L}(\alpha)\| \rightarrow \infty$ when $\|\alpha\| \rightarrow \infty$, since $\lim _{\|z\| \rightarrow \infty}\|\mathscr{I}(z)\|=\infty$ and $\lim _{\|z\| \rightarrow \infty}\|H(z)\|=\infty$. Therefore, $\mathscr{L}$ is a homeomorphism of $\mathbb{R}^{2 n}$ onto itself. Then $H=\mathscr{I}^{-1} \circ \mathscr{L} \circ \mathscr{I}$ is a homeomorphism of $\mathbb{C}^{n}$ onto itself. The proof iscompleted.
Lemma 7. For any $a, b \in \mathbb{C}^{n}$, if $P \in \mathbb{C}^{n \times n}$ is a positive definite Hermitian matrix, then $a^{*} b+b^{*} a \leq a^{*} P a+b^{*} P^{-1} b$.

Proof. Since $P$ is a positive definite Hermitian matrix, there exists an invertible matrix $Q \in \mathbb{C}^{n \times n}$, such that $P=Q^{*} Q$. For any $a, b \in \mathbb{C}^{n}$, it follows from Cauchy inequality that

$$
\begin{aligned}
& a^{*} b+b^{*} a \\
& =(Q a)^{*}\left[\left(Q^{*}\right)^{-1} b\right]+\left[\left(Q^{*}\right)^{-1} b\right]^{*}(Q a) \\
& =2 \operatorname{Re}\left\{(Q a)^{*}\left[\left(Q^{*}\right)^{-1} b\right]\right\} \leq 2\left\|(Q a)^{*}\left[\left(Q^{*}\right)^{-1} b\right]\right\| \\
& \leq 2\|Q a\|\left\|\left(Q^{*}\right)^{-1} b\right\| \leq(Q a)^{*}(Q a)+\left[\left(Q^{*}\right)^{-1} b\right]^{*}\left[\left(Q^{*}\right)^{-1} b\right] \\
& =a^{*} P a+b^{*} P^{-1} b .
\end{aligned}
$$

The proof is completed.

Lemma 8 (see [33]). For any constant matrix $W \in \mathbb{C}^{n \times n}$ and $W>0$, a scalar function $\omega:[a, b] \rightarrow \mathbb{C}^{n}$ with scalars $a<b$ such that the integration concerned are well defined, then

$$
\begin{aligned}
& (b-a) \int_{a}^{b} \omega^{*}(s) W \omega(s) \mathrm{d} s \\
& \leq\left(\int_{a}^{b} \omega(s) \mathrm{d} s\right)^{*} W\left(\int_{a}^{b} \omega(s) \mathrm{d} s\right) .
\end{aligned}
$$

Lemma 9. A given Hermitian matrix

$$
S=\left(\begin{array}{ll}
S_{11} & S_{12} \\
S_{21} & S_{22}
\end{array}\right)<0
$$

where $S_{11}^{*}=S_{11}, S_{12}^{*}=S_{21}$ and $S_{22}^{*}=S_{22}$, is equivalent to any one of the following conditions:

$$
\begin{aligned}
& \text { (1) } S_{22}<0 \text { and } S_{11}-S_{12} S_{22}^{-1} S_{21}<0 \text {, } \\
& \text { (2) } S_{11}<0 \text { and } S_{22}-S_{21} S_{11}^{-1} S_{12}<0 \text {. }
\end{aligned}
$$

Proof. (1) Note that

$$
\begin{aligned}
S & =\left(\begin{array}{ll}
S_{11} & S_{12} \\
S_{21} & S_{22}
\end{array}\right) \\
& =\left(\begin{array}{cc}
I & S_{12} S_{22}^{-1} \\
0 & I
\end{array}\right)\left(\begin{array}{cc}
S_{11}-S_{12} S_{22}^{-1} S_{21} & 0 \\
0 & S_{22}
\end{array}\right)\left(\begin{array}{cc}
I & S_{12} S_{22}^{-1} \\
0 & I
\end{array}\right)^{*} .
\end{aligned}
$$

Therefore, $S<0 \Leftrightarrow S_{22}<0$ and $S_{11}-S_{12} S_{22}^{-1} S_{21}<0$.

(2) Note that

$$
\begin{aligned}
S & =\left(\begin{array}{ll}
S_{11} & S_{12} \\
S_{21} & S_{22}
\end{array}\right) \\
& =\left(\begin{array}{cc}
I & 0 \\
S_{21} S_{11}^{-1} & I
\end{array}\right)\left(\begin{array}{cc}
S_{11} & 0 \\
0 & S_{22}-S_{21} S_{11}^{-1} S_{12}
\end{array}\right)\left(\begin{array}{cc}
I & 0 \\
S_{21} S_{11}^{-1} & I
\end{array}\right)^{*} .
\end{aligned}
$$

Therefore, $S<0 \Leftrightarrow S_{11}<0$ and $S_{22}-S_{21} S_{11}^{-1} S_{12}<0$. The proof iscompleted. 


\section{Existence and Uniqueness of Equilibrium Point}

Now we study the existence and uniqueness of the equilibrium point of system (1). As usual, we denote an equilibrium point of the system (1) by the constant complex vector $\widehat{z} \in \mathbb{C}^{n}$, where $\widehat{z}$ satisfies

$$
\begin{gathered}
-D \widehat{z}+A f(\widehat{z})+B f(\widehat{z})+\tau_{2} C f(\widehat{z})+h=0, \\
J_{k}(\widehat{z}, \widehat{z})=0, \quad k \in \mathbb{Z}^{+} .
\end{gathered}
$$

In this paper, it is assumed that the constant complex vector $\widehat{z}$ satisfies $J_{k}(\widehat{z}, \widehat{z})=0$ for $k \in \mathbb{Z}^{+}$, if $\widehat{z}$ satisfies $-D \widehat{z}+A f(\widehat{z})+$ $B f(\widehat{z})+\tau_{2} C f(\widehat{z})+h=0$. Hence, to prove the existence and uniqueness of a solution of (13), it suffices to show that the following map $\mathscr{H}: \mathbb{C}^{n} \rightarrow \mathbb{C}^{n}$ has a unique zero point:

$$
\mathscr{H}(z)=-D z+\left(A+B+\tau_{2} C\right) f(z)+h
$$

In the following, we will give some conditions for checking that $\mathscr{H}$ is a homeomorphism on $\mathbb{C}^{n}$, that is, for assuring the existence and uniqueness of the equilibrium point of system (1).

Theorem 10. Under condition (H1), the system (1) has a unique equilibrium point, if there exist two complex matrices $U_{1}, U_{2}$ and a real positive diagonal matrix $R$, such that the following LMI holds:

$$
\left(\begin{array}{cc}
\Pi_{11} & \Pi_{12} \\
\star & \Pi_{22}
\end{array}\right)<0,
$$

where $\Pi_{11}=\Gamma^{*} R \Gamma-U_{1} D-D U_{1}^{*}, \Pi_{12}=U_{1}\left(A+B+\tau_{2} C\right)-D U_{2}^{*}$, $\Pi_{22}=U_{2}\left(A+B+\tau_{2} C\right)+\left(A^{*}+B^{*}+\tau_{2} C^{*}\right) U_{2}^{*}-R$.

Proof. In the following, we will prove that $\mathscr{H}(z)$ is a homeomorphism of $\mathbb{C}^{n}$ onto itself.

First, we prove that $\mathscr{H}(z)$ is an injective map on $\mathbb{C}^{n}$. Suppose that there exist $z, z^{\prime} \in \mathbb{C}^{n}$ with $z \neq z^{\prime}$, such that $\mathscr{H}(z)=\mathscr{H}\left(z^{\prime}\right)$. Then

$$
-D\left(z-z^{\prime}\right)+\left(A+B+\tau_{2} C\right)\left(f(z)-f\left(z^{\prime}\right)\right)=0 .
$$

Multiply both sides above by $\left(z-z^{\prime}\right)^{*} U_{1}+\left(f(z)-f\left(z^{\prime}\right)\right)^{*} U_{2}$; we get

$$
\begin{aligned}
0= & -\left(z-z^{\prime}\right)^{*} U_{1} D\left(z-z^{\prime}\right) \\
& +\left(z-z^{\prime}\right)^{*} U_{1}\left(A+B+\tau_{2} C\right)\left(f(z)-f\left(z^{\prime}\right)\right) \\
& -\left(f(z)-f\left(z^{\prime}\right)\right)^{*} U_{2} D\left(z-z^{\prime}\right) \\
& +\left(f(z)-f\left(z^{\prime}\right)\right)^{*} U_{2}\left(A+B+\tau_{2} C\right)\left(f(z)-f\left(z^{\prime}\right)\right) .
\end{aligned}
$$

Taking the conjugate transpose of (17), we get that

$$
\begin{aligned}
0= & -\left(z-z^{\prime}\right)^{*} D U_{1}^{*}\left(z-z^{\prime}\right) \\
& +\left(f(z)-f\left(z^{\prime}\right)\right)^{*}\left(A^{*}+B^{*}+\tau_{2} C^{*}\right) U_{1}^{*}\left(z-z^{\prime}\right) \\
& -\left(z-z^{\prime}\right)^{*} D U_{2}^{*}\left(f(z)-f\left(z^{\prime}\right)\right)+\left(f(z)-f\left(z^{\prime}\right)\right)^{*} \\
& \times\left(A^{*}+B^{*}+\tau_{2} C^{*}\right) U_{2}^{*}\left(f(z)-f\left(z^{\prime}\right)\right) .
\end{aligned}
$$

Let $\Sigma_{1}=R-U_{2}\left(A+B+\tau_{2} C\right)-\left(A^{*}+B^{*}+\tau_{2} C^{*}\right) U_{2}^{*}$, $\Sigma_{2}=-U_{1} D-D U_{1}^{*}+\left[U_{1}\left(A+B+\tau_{2} C\right)-D U_{2}^{*}\right] \Sigma_{1}^{-1}\left[\left(A^{*}+\right.\right.$ $\left.\left.B^{*}+\tau_{2} C^{*}\right) U_{1}^{*}-U_{2} D\right]$. Then $\Sigma_{1}$ is a positive definite Hermitian matrix from Lemma 9 and LMI (15). Summing (17) and (18), applying Lemma 7, we have

$$
\begin{aligned}
& 0=-\left(z-z^{\prime}\right)^{*}\left(U_{1} D+D U_{1}^{*}\right)\left(z-z^{\prime}\right) \\
& +\left(z-z^{\prime}\right)^{*}\left[U_{1}\left(A+B+\tau_{2} C\right)-D U_{2}^{*}\right]\left(f(z)-f\left(z^{\prime}\right)\right) \\
& +\left(f(z)-f\left(z^{\prime}\right)\right)^{*} \\
& \times\left[\left(A^{*}+B^{*}+\tau_{2} C^{*}\right) U_{1}^{*}-U_{2} D\right]\left(z-z^{\prime}\right) \\
& +\left(f(z)-f\left(z^{\prime}\right)\right)^{*} \\
& \times\left[U_{2}\left(A+B+\tau_{2} C\right)+\left(A^{*}+B^{*}+\tau_{2} C^{*}\right) U_{2}^{*}\right] \\
& \times\left(f(z)-f\left(z^{\prime}\right)\right) \\
& \leq-\left(z-z^{\prime}\right)^{*}\left(U_{1} D+D U_{1}^{*}\right)\left(z-z^{\prime}\right) \\
& +\left(z-z^{\prime}\right)^{*}\left[U_{1}\left(A+B+\tau_{2} C\right)-D U_{2}^{*}\right] \\
& \times \Sigma_{1}^{-1}\left[\left(A^{*}+B^{*}+\tau_{2} C^{*}\right) U_{1}^{*}-U_{2} D\right]\left(z-z^{\prime}\right) \\
& +\left(f(z)-f\left(z^{\prime}\right)\right)^{*} \Sigma_{1}\left(f(z)-f\left(z^{\prime}\right)\right) \\
& +\left(f(z)-f\left(z^{\prime}\right)\right)^{*} \\
& \times\left[U_{2}\left(A+B+\tau_{2} C\right)+\left(A^{*}+B^{*}+\tau_{2} C^{*}\right) U_{2}^{*}\right] \\
& \times\left(f(z)-f\left(z^{\prime}\right)\right) \\
& =\left(z-z^{\prime}\right)^{*} \Sigma_{2}\left(z-z^{\prime}\right) \\
& +\left(f(z)-f\left(z^{\prime}\right)\right)^{*} R\left(f(z)-f\left(z^{\prime}\right)\right) .
\end{aligned}
$$

Since $R$ is a positive diagonal matrix, from condition (H1), we can get

$$
\begin{gathered}
\left(f(z)-f\left(z^{\prime}\right)\right)^{*} R\left(f(z)-f\left(z^{\prime}\right)\right) \\
\leq\left(z-z^{\prime}\right)^{*} \Gamma^{*} R \Gamma\left(z-z^{\prime}\right) .
\end{gathered}
$$

It follows from (19) and (20) that

$$
0 \leq\left(z-z^{\prime}\right)^{*} \Sigma_{3}\left(z-z^{\prime}\right),
$$


where $\Sigma_{3}=\Gamma^{*} R \Gamma+\Sigma_{2}=\Gamma^{*} R \Gamma-U_{1} D-D U_{1}^{*}+\left[U_{1}(A+B+\right.$ $\left.\left.\tau_{2} C\right)-D U_{2}^{*}\right] \Sigma_{1}^{-1}\left[\left(A^{*}+B^{*}+\tau_{2} C^{*}\right) U_{1}^{*}-U_{2} D\right]$. From Lemma 9 and LMI (15), we can know $\Sigma_{3}<0$. Then $z-z^{\prime}=0$ from (21). Therefore, $\mathscr{H}(z)$ is an injective map on $\mathbb{C}^{n}$.

Second, we prove $\|\mathscr{H}(z)\| \rightarrow \infty$ as $\|z\| \rightarrow \infty$. Let $\widetilde{\mathscr{H}}(z)=\mathscr{H}(z)-\mathscr{H}(0)$. By Lemma 7 , we have

$$
\begin{aligned}
{\left[z^{*} U_{1}\right.} & \left.+(f(z)-f(0))^{*} U_{2}\right] \widetilde{\mathscr{H}}(z) \\
& +\widetilde{\mathscr{H}}(z)^{*}\left[z^{*} U_{1}+(f(z)-f(0))^{*} U_{2}\right]^{*} \\
= & -z^{*}\left(U_{1} D+D U_{1}^{*}\right) z \\
& +z^{*}\left[U_{1}\left(A+B+\tau_{2} C\right)-D U_{2}^{*}\right](f(z)-f(0)) \\
& +(f(z)-f(0))^{*}\left[\left(A^{*}+B^{*}+\tau_{2} C^{*}\right) U_{1}^{*}-U_{2} D\right] z \\
& +(f(z)-f(0))^{*} \\
& \times\left[U_{2}\left(A+B+\tau_{2} C\right)+\left(A^{*}+B^{*}+\tau_{2} C^{*}\right) U_{2}^{*}\right] \\
& \times(f(z)-f(0)) \\
\leq & -z^{*}\left(U_{1} D+D U_{1}^{*}\right) z \\
& +z^{*}\left[U_{1}\left(A+B+\tau_{2} C\right)-D U_{2}^{*}\right] \\
& \times \Sigma_{1}^{-1}\left[\left(A^{*}+B^{*}+\tau_{2} C^{*}\right) U_{1}^{*}-U_{2} D\right] z \\
& +(f(z)-f(0))^{*} \Sigma_{1}(f(z)-f(0)) \\
& +(f(z)-f(0))^{*} \\
& \times\left[U_{2}\left(A+B+\tau_{2} C\right)+\left(A^{*}+B^{*}+\tau_{2} C^{*}\right) U_{2}^{*}\right] \\
& \times(f(z)-f(0)) \\
= & z^{*} \Sigma_{2} z+(f(z)-f(0))^{*} R(f(z)-f(0)) .
\end{aligned}
$$

It follows from condition ( $\mathrm{H} 1)$ that

$$
(f(z)-f(0))^{*} R(f(z)-f(0)) \leq z^{*} \Gamma^{*} R \Gamma z .
$$

Then from (22), (23), and $\Sigma_{3}=\Gamma^{*} R \Gamma+\Sigma_{2}<0$, we obtain

$$
\begin{aligned}
{\left[z^{*} U_{1}\right.} & \left.+(f(z)-f(0))^{*} U_{2}\right] \widetilde{\mathscr{H}}(z) \\
& +\widetilde{\mathscr{H}}(z)^{*}\left[z^{*} U_{1}+(f(z)-f(0))^{*} U_{2}\right]^{*} \\
\leq & z^{*} \Sigma_{3} z \leq-\rho_{\min }\left(-\Sigma_{3}\right)\|z\|^{2},
\end{aligned}
$$

which can imply that

$$
\begin{aligned}
\lambda_{\min }\left(-\Sigma_{3}\right)\|z\|^{2} & \leq 2\left\|\left[z^{*} U_{1}+(f(z)-f(0))^{*} U_{2}\right]\right\|\|\widetilde{\mathscr{H}}(z)\| \\
& \leq 2\left(\left\|U_{1}\right\|+\left\|\Gamma^{*} U_{2}\right\|\right)\|z\|\|\widetilde{\mathscr{H}}(z)\| .
\end{aligned}
$$

When $z \neq 0$, we have

$$
\|\widetilde{\mathscr{H}}(z)\| \geq \frac{\lambda_{\min }\left(-\Sigma_{3}\right)\|z\|}{2\left(\left\|U_{1}\right\|+\left\|\Gamma^{*} U_{2}\right\|\right)} .
$$

Therefore, $\|\widetilde{\mathscr{H}}(z)\| \rightarrow \infty$ as $\|z\| \rightarrow \infty$ which implies $\|\mathscr{H}(z)\| \rightarrow \infty$ as $\|z\| \rightarrow \infty$. From Lemma 6 , we know that $\mathscr{H}(z)$ is a homeomorphism of $\mathbb{C}^{n}$. Thus, the system (1) has a unique equilibrium point.

Remark 11. It should be noted that Theorem 10 is independent of leakage time delay and initial conditions. So, the time delays in the leakage terms do not affect the existence and uniqueness of the equilibrium point.

\section{Global $\mu$-Stability Results}

In the preceding section, we have shown the existence and uniqueness of the equilibrium point for system (1). In this section, we will further investigate the global $\mu$-stability of the unique equilibrium point. For this purpose, the impulsive function $J_{k}$ which is viewed as a perturbation of the equilibrium point $\widehat{z}$ of system (1) without impulses is defined by

$$
J_{k}\left(z\left(t_{k}^{-}\right), z_{t_{k}^{-}}\right)=E_{k}\left[z\left(t_{k}^{-}\right)-\widehat{z}-D \int_{t_{k}-\delta}^{t_{k}}(z(s)-\widehat{z}) \mathrm{d} s\right],
$$

where $k \in \mathbb{Z}^{+}, E_{k} \in \mathbb{C}^{n \times n}$. It is obvious that $J_{k}(\widehat{z}, \widehat{z})=0$.

Remark 12. The type of impulse such as (27) describes the fact that the instantaneous perturbations are not only related to the state of neurons at impulse times $t_{k}$ but also related to the state of neurons in recent history, which reflects more realistic dynamics [19].

Theorem 13. Under the conditions of Theorem 10, the equilibrium point of system (1) is globally $\mu$-stable, if there exist six positive definite Hermitian matrices $P_{1}, P_{2}, P_{3}, P_{4}, P_{5}$, and $P_{6}$, two real positive diagonal matrices $R_{1}$ and $R_{2}$, three complex matrices $Q_{1}, Q_{2}$, and $Q_{3}$, a positive differential function $\mu(t)$, and three constants $\alpha \geq 0, \beta>0$, and $T>0$ such that

$$
\begin{gathered}
0 \leq \frac{\dot{\mu}(t)}{\mu(t)} \leq \alpha, \\
\frac{\min \left\{\mu(t-\delta), \mu\left(t-\tau_{1}\right), \mu\left(t-\tau_{2}\right)\right\}}{\mu(t)} \geq \beta, \\
\forall t \in[T, \infty),
\end{gathered}
$$


and the following LMIs hold:

$$
\left(\begin{array}{cccccccc}
\Omega_{11} & \Omega_{12} & P_{1} D & \frac{\beta^{2}}{\tau_{1}} P_{5} & \Omega_{15} & 0 & 0 & 0 \\
\star & \Omega_{22} & Q_{1}^{*} D & Q_{3} & -P_{1} D & -Q_{1}^{*} A & -Q_{1}^{*} B & -Q_{1}^{*} C \\
\star & \star & \Omega_{33} & D Q_{3} & -D P_{1} D & -Q_{2}^{*} A & -Q_{2}^{*} B & -Q_{2}^{*} C \\
\star & \star & \star & \Omega_{44} & 0 & -Q_{3}^{*} A & -Q_{3}^{*} B & -Q_{3}^{*} C \\
\star & \star & \star & \star & -\frac{\beta^{2}}{\delta} P_{3} & 0 & 0 & 0 \\
\star & \star & \star & \star & \star & \Omega_{66} & 0 & 0 \\
\star & \star & \star & \star & \star & \star & -R_{2} & 0 \\
\star & \star & \star & \star & \star & \star & \star & -\frac{\beta^{2}}{\tau_{2}} P_{6}
\end{array}\right)<0,
$$

$$
\left(\begin{array}{cc}
P_{1} & \left(I+E_{k}\right) P_{1} \\
\star & P_{1}
\end{array}\right)>0,
$$

where $\Omega_{11}=2 \alpha P_{1}-D P_{1}-P_{1} D+2 \alpha P_{2}+\delta P_{3}+P_{4}-\left(\beta^{2} / \tau_{1}\right) P_{5}+$ $R_{1} \Gamma, \Omega_{12}=P_{1}+P_{2}, \Omega_{15}=D P_{1} D-2 \alpha P_{1} D, \Omega_{22}=Q_{1}^{*}+Q_{1}+$ $\tau_{1} P_{5}, \Omega_{33}=Q_{2}^{*} D+D Q_{2}, \Omega_{44}=-\beta^{2} P_{4}-\left(\beta^{2} / \tau_{1}\right) P_{5}+R_{2} \Gamma$, and $\Omega_{66}=\tau_{2} P_{6}-R_{1}$.

Proof. Under the condition of Theorem 10, system (1) has a unique equilibrium point $\widehat{z}$. Then we shift the equilibrium point of (1) to the origin by the translation $\widetilde{z}(t)=z(t)-\widehat{z}$ and obtain

$$
\begin{aligned}
\dot{\tilde{z}}(t)= & -D \widetilde{z}(t-\delta)+A g(\widetilde{z}(t))+B g\left(\widetilde{z}\left(t-\tau_{1}\right)\right) \\
& +C \int_{t-\tau_{2}}^{t} g(\widetilde{z}(s)) \mathrm{d} s, \quad t \neq t_{k}, t>0, \\
\Delta \widetilde{z}\left(t_{k}\right)= & \widetilde{z}\left(t_{k}\right)-\widetilde{z}\left(t_{k}^{-}\right) \\
= & E_{k}\left[\widetilde{z}\left(t_{k}^{-}\right)-D \int_{t_{k}-\delta}^{t_{k}} \widetilde{z}(s) \mathrm{d} s\right], \quad k \in \mathbb{Z}^{+} .
\end{aligned}
$$

Consider the following Lyapunov-Krasovskii functional candidate:

$$
V(t)=V_{1}(t)+V_{2}(t)+V_{3}(t)+V_{4}(t)
$$

where

$$
\begin{aligned}
V_{1}(t)= & \mu^{2}(t)\left[\tilde{z}(t)-D \int_{t-\delta}^{t} \widetilde{z}(s) \mathrm{d} s\right]^{*} \\
& \times P_{1}\left[\widetilde{z}(t)-D \int_{t-\delta}^{t} \widetilde{z}(s) \mathrm{d} s\right], \\
V_{2}(t)= & \mu^{2}(t) \widetilde{z}^{*}(t) P_{2} \widetilde{z}(t) \\
& +\int_{0}^{\delta} \int_{t-u}^{t} \mu^{2}(s) \widetilde{z}^{*}(s) P_{3} \widetilde{z}(s) \mathrm{d} s \mathrm{~d} u,
\end{aligned}
$$

$$
\begin{gathered}
V_{3}(t)=\int_{t-\tau_{1}}^{t} \mu^{2}(s) \widetilde{z}^{*}(s) P_{4} \tilde{z}(s) \mathrm{d} s \\
\quad+\int_{0}^{\tau_{1}} \int_{t-u}^{t} \mu^{2}(s) \dot{\tilde{z}}^{*}(s) P_{5} \dot{\tilde{z}}(s) \mathrm{d} s \mathrm{~d} u, \\
V_{4}(t)=\int_{0}^{\tau_{2}} \int_{t-u}^{t} \mu^{2}(s) g^{*}(\widetilde{z}(s)) P_{6} g(\widetilde{z}(s)) \mathrm{d} s \mathrm{~d} u .
\end{gathered}
$$

Calculating the upper right derivative of $V$ along the solution of (31), applying Lemma 8, we get

$$
\begin{aligned}
& D^{+} V_{1}(t) \\
& =2 \mu(t) \dot{\mu}(t)\left[\widetilde{z}(t)-D \int_{t-\delta}^{t} \widetilde{z}(s) \mathrm{d} s\right]^{*} \\
& \times P_{1}\left[\tilde{z}(t)-D \int_{t-\delta}^{t} \widetilde{z}(s) \mathrm{d} s\right] \\
& +\mu^{2}(t)[\dot{\tilde{z}}(t)-D \widetilde{z}(t)+D \widetilde{z}(t-\delta)]^{*} \\
& \times P_{1}\left[\tilde{z}(t)-D \int_{t-\delta}^{t} \tilde{z}(s) \mathrm{d} s\right] \\
& +\mu^{2}(t)\left[\widetilde{z}(t)-D \int_{t-\delta}^{t} \widetilde{z}(s) \mathrm{d} s\right]^{*} \\
& \times P_{1}[\dot{\widetilde{z}}(t)-D \widetilde{z}(t)+D \widetilde{z}(t-\delta)] \\
& \leq \mu^{2}(t)\left[2 \alpha \widetilde{z}^{*}(t) P_{1} \widetilde{z}(t)-2 \alpha \widetilde{z}^{*}(t) P_{1} D \int_{t-\delta}^{t} \widetilde{z}(s) \mathrm{d} s\right. \\
& -2 \alpha \int_{t-\delta}^{t} \widetilde{z}^{*}(s) \mathrm{d} s D P_{1} \widetilde{z}(t) \mathrm{d} s \\
& +2 \alpha \int_{t-\delta}^{t} \widetilde{z}^{*}(s) \mathrm{d} s D P_{1} D \int_{t-\delta}^{t} \tilde{z}(s) \mathrm{d} s
\end{aligned}
$$




$$
\begin{aligned}
& +\dot{\bar{z}}^{*}(t) P_{1} \widetilde{z}(t)-\dot{\bar{z}}^{*}(t) P_{1} D \int_{t-\delta}^{t} \widetilde{z}(s) \mathrm{d} s \\
& -\widetilde{z}^{*}(t) D P_{1} \widetilde{z}(t)+\widetilde{z}^{*}(t) D P_{1} D \int_{t-\delta}^{t} \widetilde{z}(s) \mathrm{d} s \\
& +\widetilde{z}^{*}(t-\delta) D P_{1} \widetilde{z}(t) \\
& -\widetilde{z}^{*}(t-\delta) D P_{1} D \int_{t-\delta}^{t} \tilde{z}(s) \mathrm{d} s+\widetilde{z}^{*}(t) P_{1} \dot{\tilde{z}}(t) \\
& -\int_{t-\delta}^{t} \widetilde{z}^{*}(s) \mathrm{d} s D P_{1} \dot{\tilde{z}}(t) \\
& -\widetilde{z}^{*}(t) P_{1} D \widetilde{z}(t)+\int_{t-\delta}^{t} \widetilde{z}^{*}(s) \mathrm{d} s D P_{1} D \widetilde{z}(t) \\
& +\widetilde{z}^{*}(t) P_{1} D \widetilde{z}(t-\delta) \\
& \left.-\int_{t-\delta}^{t} \widetilde{z}^{*}(s) \mathrm{d} s D P_{1} D \widetilde{z}(t-\delta)\right] \\
& =\mu^{2}(t)\left[\widetilde{z}^{*}(t)\left(2 \alpha P_{1}-D P_{1}-P_{1} D\right) \widetilde{z}(t)\right. \\
& +\widetilde{z}^{*}(t)\left(D P_{1} D-2 \alpha P_{1} D\right) \int_{t-\delta}^{t} \tilde{z}(s) \mathrm{d} s \\
& +\int_{t-\delta}^{t} \widetilde{z}^{*}(s) \mathrm{d} s\left(D P_{1} D-2 \alpha D P_{1}\right) \widetilde{z}(t) \\
& +2 \alpha \int_{t-\delta}^{t} \widetilde{z}^{*}(s) \mathrm{d} s D P_{1} D \int_{t-\delta}^{t} \widetilde{z}(s) \mathrm{d} s \\
& +\dot{\widetilde{z}}^{*}(t) P_{1} \widetilde{z}(t)+\widetilde{z}^{*}(t) P_{1} \dot{\tilde{z}}(t) \\
& -\dot{\bar{z}}^{*}(t) P_{1} D \int_{t-\delta}^{t} \tilde{z}(s) \mathrm{d} s-\int_{t-\delta}^{t} \widetilde{z}^{*}(s) \mathrm{d} s D P_{1} \dot{\tilde{z}}(t) \\
& +\widetilde{z}^{*}(t-\delta) D P_{1} \widetilde{z}(t)+\widetilde{z}^{*}(t) P_{1} D \widetilde{z}(t-\delta) \\
& -\widetilde{z}^{*}(t-\delta) D P_{1} D \int_{t-\delta}^{t} \tilde{z}(s) \mathrm{d} s \\
& \left.-\int_{t-\delta}^{t} \widetilde{z}^{*}(s) \mathrm{d} s D P_{1} D \widetilde{z}(t-\delta)\right] \\
& +\delta \mu^{2}(t) \widetilde{z}^{*}(t) P_{3} \widetilde{z}(t) \\
& -\int_{t-\delta}^{t} \mu^{2}(s) \widetilde{z}^{*}(s) P_{3} \widetilde{z}(s) \mathrm{d} s \\
& \leq 2 \alpha \mu^{2}(t) \widetilde{z}^{*}(t) P_{2} \widetilde{z}(t)+\mu^{2}(t) \dot{\bar{z}}^{*}(t) P_{2} \widetilde{z}(t) \\
& +\mu^{2}(t) \widetilde{z}^{*}(t) P_{2} \dot{\tilde{z}}(t) \\
& +\delta \mu^{2}(t) \widetilde{z}^{*}(t) P_{3} \widetilde{z}(t) \\
& -\beta^{2} \mu^{2}(t) \int_{t-\delta}^{t} \widetilde{z}^{*}(s) P_{3} \widetilde{z}(s) \mathrm{d} s \\
& \leq \mu^{2}(t)\left[\widetilde{z}^{*}(t)\left(2 \alpha P_{2}+\delta P_{3}\right) \widetilde{z}(t)+\widetilde{z}^{*}(t) P_{2} \dot{\widetilde{z}}(t)\right. \\
& +\dot{\bar{z}}^{*}(t) P_{2} \widetilde{z}(t) \\
& \left.-\frac{\beta^{2}}{\delta} \int_{t-\delta}^{t} \widetilde{z}^{*}(s) \mathrm{d} s P_{3} \int_{t-\delta}^{t} \tilde{z}(s) \mathrm{d} s\right] \\
& D^{+} V_{3}(t) \\
& =\mu^{2}(t) \widetilde{z}^{*}(t) P_{4} \widetilde{z}(t) \\
& -\mu^{2}\left(t-\tau_{1}\right) \widetilde{z}^{*}\left(t-\tau_{1}\right) P_{4} \tilde{z}\left(t-\tau_{1}\right) \\
& +\tau_{1} \mu^{2}(t) \dot{\bar{z}}^{*}(t) P_{5} \dot{\widetilde{z}}(t) \\
& -\int_{0}^{\tau_{1}} \mu^{2}(t-u) \dot{\vec{z}}^{*}(t-u) P_{5} \dot{\tilde{z}}(t-u) \mathrm{d} u \\
& =\mu^{2}(t) \widetilde{z}^{*}(t) P_{4} \widetilde{z}(t) \\
& -\mu^{2}\left(t-\tau_{1}\right) \widetilde{z}^{*}\left(t-\tau_{1}\right) P_{4} \tilde{z}\left(t-\tau_{1}\right) \\
& +\tau_{1} \mu^{2}(t) \dot{\bar{z}}^{*}(t) P_{5} \dot{\widetilde{z}}(t) \\
& -\int_{t-\tau_{1}}^{t} \mu^{2}(s) \dot{\bar{z}}^{*}(s) P_{5} \dot{\tilde{z}}(s) \mathrm{d} s \\
& \leq \mu^{2}(t)\left[\widetilde{z}^{*}(t) P_{4} \widetilde{z}(t)-\beta^{2} \widetilde{z}^{*}\left(t-\tau_{1}\right) P_{4} \widetilde{z}\left(t-\tau_{1}\right)\right. \\
& \begin{array}{l}
+\tau_{1} \dot{\bar{z}}^{*}(t) P_{5} \dot{\tilde{z}}(t) \\
\left.\quad-\frac{\beta^{2}}{\tau_{1}} \int_{t-\tau_{1}}^{t} \dot{\tilde{z}}^{*}(s) \mathrm{d} s P_{5} \int_{t-\tau_{1}}^{t} \dot{\tilde{z}}(s) \mathrm{d} s\right] \\
=\mu^{2}(t)\left[\widetilde{z}^{*}(t) P_{4} \tilde{z}(t)-\beta^{2} \widetilde{z}^{*}\left(t-\tau_{1}\right) P_{4} \widetilde{z}\left(t-\tau_{1}\right)\right. \\
+\tau_{1} \dot{\bar{z}}^{*}(t) P_{5} \dot{\tilde{z}}(t)-\frac{\beta^{2}}{\tau_{1}}\left(\widetilde{z}^{*}(t)-\widetilde{z}^{*}\left(t-\tau_{1}\right)\right) \\
\left.\quad \times P_{5}\left(\widetilde{z}(t)-\widetilde{z}\left(t-\tau_{1}\right)\right)\right]
\end{array} \\
& \begin{array}{l}
+\tau_{1} \dot{\bar{z}}^{*}(t) P_{5} \dot{\tilde{z}}(t) \\
\left.\quad-\frac{\beta^{2}}{\tau_{1}} \int_{t-\tau_{1}}^{t} \dot{\tilde{z}}^{*}(s) \mathrm{d} s P_{5} \int_{t-\tau_{1}}^{t} \dot{\tilde{z}}(s) \mathrm{d} s\right] \\
=\mu^{2}(t)\left[\widetilde{z}^{*}(t) P_{4} \tilde{z}(t)-\beta^{2} \widetilde{z}^{*}\left(t-\tau_{1}\right) P_{4} \widetilde{z}\left(t-\tau_{1}\right)\right. \\
+\tau_{1} \dot{\bar{z}}^{*}(t) P_{5} \dot{\tilde{z}}(t)-\frac{\beta^{2}}{\tau_{1}}\left(\widetilde{z}^{*}(t)-\widetilde{z}^{*}\left(t-\tau_{1}\right)\right) \\
\left.\quad \times P_{5}\left(\widetilde{z}(t)-\widetilde{z}\left(t-\tau_{1}\right)\right)\right]
\end{array} \\
& \begin{array}{l}
+\tau_{1} \dot{\bar{z}}^{*}(t) P_{5} \dot{\bar{z}}(t) \\
\left.\quad-\frac{\beta^{2}}{\tau_{1}} \int_{t-\tau_{1}}^{t} \dot{\dot{z}}^{*}(s) \mathrm{d} s P_{5} \int_{t-\tau_{1}}^{t} \dot{\tilde{z}}(s) \mathrm{d} s\right] \\
=\mu^{2}(t)\left[\widetilde{z}^{*}(t) P_{4} \widetilde{z}(t)-\beta^{2} \widetilde{z}^{*}\left(t-\tau_{1}\right) P_{4} \widetilde{z}\left(t-\tau_{1}\right)\right. \\
+\tau_{1} \dot{\bar{z}}^{*}(t) P_{5} \dot{\bar{z}}(t)-\frac{\beta^{2}}{\tau_{1}}\left(\widetilde{z}^{*}(t)-\widetilde{z}^{*}\left(t-\tau_{1}\right)\right) \\
\left.\quad \times P_{5}\left(\widetilde{z}(t)-\widetilde{z}\left(t-\tau_{1}\right)\right)\right]
\end{array} \\
& \begin{array}{l}
+\tau_{1} \dot{\bar{z}}^{*}(t) P_{5} \dot{\tilde{z}}(t) \\
\left.\quad-\frac{\beta^{2}}{\tau_{1}} \int_{t-\tau_{1}}^{t} \dot{\tilde{z}}^{*}(s) \mathrm{d} s P_{5} \int_{t-\tau_{1}}^{t} \dot{\tilde{z}}(s) \mathrm{d} s\right] \\
=\mu^{2}(t)\left[\widetilde{z}^{*}(t) P_{4} \tilde{z}(t)-\beta^{2} \widetilde{z}^{*}\left(t-\tau_{1}\right) P_{4} \widetilde{z}\left(t-\tau_{1}\right)\right. \\
+\tau_{1} \dot{\bar{z}}^{*}(t) P_{5} \dot{\tilde{z}}(t)-\frac{\beta^{2}}{\tau_{1}}\left(\widetilde{z}^{*}(t)-\widetilde{z}^{*}\left(t-\tau_{1}\right)\right) \\
\left.\quad \times P_{5}\left(\widetilde{z}(t)-\widetilde{z}\left(t-\tau_{1}\right)\right)\right]
\end{array} \\
& \begin{array}{l}
+\tau_{1} \dot{\bar{z}}^{*}(t) P_{5} \dot{\tilde{z}}(t) \\
\left.\quad-\frac{\beta^{2}}{\tau_{1}} \int_{t-\tau_{1}}^{t} \dot{\tilde{z}}^{*}(s) \mathrm{d} s P_{5} \int_{t-\tau_{1}}^{t} \dot{\tilde{z}}(s) \mathrm{d} s\right] \\
=\mu^{2}(t)\left[\widetilde{z}^{*}(t) P_{4} \tilde{z}(t)-\beta^{2} \widetilde{z}^{*}\left(t-\tau_{1}\right) P_{4} \widetilde{z}\left(t-\tau_{1}\right)\right. \\
+\tau_{1} \dot{\bar{z}}^{*}(t) P_{5} \dot{\tilde{z}}(t)-\frac{\beta^{2}}{\tau_{1}}\left(\widetilde{z}^{*}(t)-\widetilde{z}^{*}\left(t-\tau_{1}\right)\right) \\
\left.\quad \times P_{5}\left(\widetilde{z}(t)-\widetilde{z}\left(t-\tau_{1}\right)\right)\right]
\end{array} \\
& =2 \mu(t) \dot{\mu}(t) \widetilde{z}^{*}(t) P_{2} \widetilde{z}(t)+\mu^{2}(t) \dot{\tilde{z}}^{*}(t) P_{2} \widetilde{z}(t) \\
& +\mu^{2}(t) \widetilde{z}^{*}(t) P_{2} \dot{\tilde{z}}(t) \\
& +\delta \mu^{2}(t) \widetilde{z}^{*}(t) P_{3} \widetilde{z}(t) \\
& -\int_{0}^{\delta} \mu^{2}(t-u) \widetilde{z}^{*}(t-u) P_{3} \widetilde{z}(t-u) \mathrm{d} u \\
& \leq 2 \alpha \mu^{2}(t) \widetilde{z}^{*}(t) P_{2} \widetilde{z}(t) \\
& +\mu^{2}(t) \dot{\vec{z}}^{*}(t) P_{2} \widetilde{z}(t)+\mu^{2}(t) \widetilde{z}^{*}(t) P_{2} \dot{\tilde{z}}(t)
\end{aligned}
$$




$$
\begin{aligned}
D^{+} V_{4}(t) & \\
= & \tau_{2} \mu^{2}(t) g^{*}(\widetilde{z}(t)) P_{6} g(\widetilde{z}(t)) \\
& -\int_{0}^{\tau_{2}} \mu^{2}(t-u) g^{*}(\widetilde{z}(t-u)) P_{6} g(\widetilde{z}(t-u)) \mathrm{d} u \\
= & \tau_{2} \mu^{2}(t) g^{*}(\widetilde{z}(t)) P_{6} g(\widetilde{z}(t)) \\
& -\int_{t-\tau_{2}}^{t} \mu^{2}(s) g^{*}(\widetilde{z}(s)) P_{6} g(\widetilde{z}(s)) \mathrm{d} s \\
\leq & \tau_{2} \mu^{2}(t) g^{*}(\widetilde{z}(t)) P_{6} g(\widetilde{z}(t)) \\
& -\beta^{2} \mu^{2}(t) \int_{t-\tau_{2}}^{t} g^{*}(\widetilde{z}(s)) P_{6} g(\widetilde{z}(s)) \mathrm{d} s \\
\leq & \mu^{2}(t)\left[\tau_{2} g^{*}(\widetilde{z}(t)) P_{6} g(\widetilde{z}(t))\right. \\
& \left.\quad-\frac{\beta^{2}}{\tau_{2}} \int_{t-\tau_{2}}^{t} g^{*}(\widetilde{z}(s)) \mathrm{d} s P_{6} \int_{t-\tau_{2}}^{t} g(\widetilde{z}(s)) \mathrm{d} s\right] .
\end{aligned}
$$

Combining (34), (35), (36), and (37), we can deduce that

$$
\begin{aligned}
& D^{+} V(t) \\
& \leq \mu^{2}(t)\left[\widetilde { z } ^ { * } ( t ) \left(2 \alpha P_{1}-D P_{1}-P_{1} D+2 \alpha P_{2}\right.\right. \\
& \left.+\delta P_{3}+P_{4}-\frac{\beta^{2}}{\tau_{1}} P_{5}\right) \tilde{z}(t) \\
& +\widetilde{z}^{*}(t)\left(P_{1}+P_{2}\right) \dot{\tilde{z}}(t) \\
& +\dot{\tilde{z}}^{*}(t)\left(P_{1}+P_{2}\right) \widetilde{z}(t) \\
& +\widetilde{z}^{*}(t) P_{1} D \widetilde{z}(t-\delta) \\
& +\widetilde{z}^{*}(t-\delta) D P_{1} \widetilde{z}(t) \\
& +\frac{\beta^{2}}{\tau_{1}} \widetilde{z}^{*}(t) P_{5} \widetilde{z}\left(t-\tau_{1}\right) \\
& +\frac{\beta^{2}}{\tau_{1}} \widetilde{z}^{*}\left(t-\tau_{1}\right) P_{5} \widetilde{z}(t) \\
& +\widetilde{z}^{*}(t)\left(D P_{1} D-2 \alpha P_{1} D\right) \int_{t-\delta}^{t} \widetilde{z}(s) \mathrm{d} s \\
& +\int_{t-\delta}^{t} \widetilde{z}^{*}(s) \mathrm{d} s\left(D P_{1} D-2 \alpha D P_{1}\right) \widetilde{z}(t) \\
& -\dot{\tilde{z}}^{*}(t) P_{1} D \int_{t-\delta}^{t} \tilde{z}(s) \mathrm{d} s
\end{aligned}
$$

From assumption (H1), we have

$$
\left|g_{j}\left(\widetilde{z}_{j}(t)\right)\right| \leq \gamma_{j}\left|\widetilde{z}_{j}(t)\right|
$$

for $j=1,2, \ldots, n$. Let $R_{1}=\operatorname{diag}\left(r_{1}, r_{2}, \ldots, r_{n}\right)>0$. It follows from (39) that

$$
r_{j} \mu^{2}(t) g_{j}^{*}\left(\widetilde{z}_{j}(t)\right) g_{j}\left(\widetilde{z}_{j}(t)\right)-r_{j} \mu^{2}(t) \gamma_{j}^{2} \widetilde{z}_{j}^{*}(t) \widetilde{z}_{j}(t) \leq 0
$$

for $j=1,2, \ldots, n$. Hence

$$
\mu^{2}(t) g^{*}(\widetilde{z}(t)) R_{1} g(\widetilde{z}(t))-\mu^{2}(t) \widetilde{z}^{*}(t) R_{1} \Gamma \widetilde{z}(t) \leq 0 .
$$

Also, we can get that

$$
\begin{aligned}
\mu^{2}(t) & g^{*}\left(\widetilde{z}\left(t-\tau_{1}\right)\right) R_{2} g\left(\widetilde{z}\left(t-\tau_{1}\right)\right) \\
& -\mu^{2}(t) \widetilde{z}^{*}\left(t-\tau_{1}\right) R_{2} \Gamma \widetilde{z}\left(t-\tau_{1}\right) \leq 0 .
\end{aligned}
$$

From (31), we have that

$$
\begin{aligned}
& 0=\mu^{2}(t) {\left[Q_{1} \dot{\tilde{z}}(t)+Q_{2} \tilde{z}(t-\delta)+Q_{3} \widetilde{z}\left(t-\tau_{1}\right)\right]^{*} } \\
& \times\left[\dot{\tilde{z}}(t)+D \widetilde{z}(t-\delta)-A g(\widetilde{z}(t))-B g\left(\widetilde{z}\left(t-\tau_{1}\right)\right)\right. \\
&\left.\quad-C \int_{t-\tau_{2}}^{t} g(\widetilde{z}(s)) \mathrm{d} s\right]
\end{aligned}
$$




$$
\begin{aligned}
& +\mu^{2}(t)[\dot{\tilde{z}}(t)+D \widetilde{z}(t-\delta)-A g(\widetilde{z}(t)) \\
& \left.\quad-B g\left(\widetilde{z}\left(t-\tau_{1}\right)\right)-C \int_{t-\tau_{2}}^{t} g(\widetilde{z}(s)) \mathrm{d} s\right]^{*} \\
& \times\left[Q_{1} \dot{\tilde{z}}(t)+Q_{2} \widetilde{z}(t-\delta)+Q_{3} \widetilde{z}\left(t-\tau_{1}\right)\right] .
\end{aligned}
$$

It follows from (38), (41), (42), and (43) that

$$
D^{+} V(t) \leq \mu^{2}(t) w^{*}(t) \Omega w(t)
$$

where $w(t)=\left(\widetilde{z}^{*}(t), \dot{\tilde{z}}^{*}(t), \widetilde{z}^{*}(t-\delta), \widetilde{z}(t-\right.$ $\left.\left.\tau_{1}\right), \int_{t-\delta}^{t} \widetilde{z}^{*}(s) \mathrm{d} s, g^{*}(\widetilde{z}(t)), g^{*}\left(\widetilde{z}\left(t-\tau_{1}\right)\right), \int_{t-\tau_{2}}^{t} g^{*}(\widetilde{z}(s)) \mathrm{d} s\right)^{*}$ and

$$
\Omega=\left(\begin{array}{cccccccc}
\Omega_{11} & \Omega_{12} & P_{1} D & \frac{\beta^{2}}{\tau_{1}} P_{5} & \Omega_{15} & 0 & 0 & 0 \\
\star & \Omega_{22} & Q_{1}^{*} D & Q_{3} & -P_{1} D & -Q_{1}^{*} A & -Q_{1}^{*} B & -Q_{1}^{*} C \\
\star & \star & \Omega_{33} & D Q_{3} & -D P_{1} D & -Q_{2}^{*} A & -Q_{2}^{*} B & -Q_{2}^{*} C \\
\star & \star & \star & \Omega_{44} & 0 & -Q_{3}^{*} A & -Q_{3}^{*} B & -Q_{3}^{*} C \\
\star & \star & \star & \star & -\frac{\beta^{2}}{\delta} P_{3} & 0 & 0 & 0 \\
\star & \star & \star & \star & \star & \Omega_{66} & 0 & 0 \\
\star & \star & \star & \star & \star & \star & -R_{2} & 0 \\
\star & \star & \star & \star & \star & \star & \star & -\frac{\beta^{2}}{\tau_{2}} P_{6}
\end{array}\right)
$$

with $\Omega_{11}=2 \alpha \mathrm{P}_{1}-D P_{1}-P_{1} D+2 \alpha P_{2}+\delta P_{3}+P_{4}-\left(\beta^{2} / \tau_{1}\right) P_{5}+R_{1} \Gamma$, $\Omega_{12}=P_{1}+P_{2}, \Omega_{15}=D P_{1} D-2 \alpha P_{1} D, \Omega_{22}=Q_{1}^{*}+Q_{1}+\tau_{1} P_{5}$, $\Omega_{33}=Q_{2}^{*} D+D Q_{2}, \Omega_{44}=-\beta^{2} P_{4}-\left(\beta^{2} / \tau_{1}\right) P_{5}+R_{2} \Gamma, \Omega_{66}=$ $\tau_{2} P_{6}-R_{1}$. Thus, we get from (29) and (44) that

$$
D^{+} V(t) \leq 0, \quad t \in\left[t_{k-1}, t_{k}\right) \cap[T, \infty), k \in \mathbb{Z}^{+} .
$$

In addition, we note that

$$
\begin{aligned}
&\left(\begin{array}{cc}
P_{1} & \left(I+E_{k}\right) P_{1} \\
\star & P_{1}
\end{array}\right)>0 \\
& \quad \Longleftrightarrow\left(\begin{array}{cc}
I & 0 \\
0 & P_{1}^{-1}
\end{array}\right)\left(\begin{array}{cc}
P_{1} & \left(I+E_{k}\right) P_{1} \\
\star & P_{1}
\end{array}\right)\left(\begin{array}{cc}
I & 0 \\
0 & P_{1}^{-1}
\end{array}\right)>0 \\
& \Longleftrightarrow\left(\begin{array}{cc}
P_{1} & \left(I+E_{k}\right) \\
\star & P_{1}^{-1}
\end{array}\right)>0 \\
& \Longleftrightarrow P_{1}-\left(I+E_{k}\right)^{*} P_{1}\left(I+E_{k}\right)>0
\end{aligned}
$$

in which the last equivalent relation is obtained by Lemma 9. Thus, it yields

$$
\begin{aligned}
V_{1}\left(t_{k}\right)= & \mu^{2}\left(t_{k}\right)\left[\widetilde{z}\left(t_{k}\right)-D \int_{t_{k}-\delta}^{t_{k}} \tilde{z}(s) \mathrm{d} s\right]^{*} \\
& \times P_{1}\left[\widetilde{z}\left(t_{k}\right)-D \int_{t_{k}-\delta}^{t_{k}} \widetilde{z}(s) \mathrm{d} s\right] \\
= & \mu^{2}\left(t_{k}^{-}\right)\left[\widetilde{z}\left(t_{k}^{-}\right)-D \int_{t_{k}-\delta}^{t_{k}} \widetilde{z}(s) \mathrm{d} s\right]^{*}
\end{aligned}
$$

$$
\begin{aligned}
& \times\left(I+E_{k}\right)^{*} P_{1}\left(I+E_{k}\right) \\
& \times\left[\widetilde{z}\left(t_{k}\right)-D \int_{t_{k}-\delta}^{t_{k}} \widetilde{z}(s) \mathrm{d} s\right] \\
\leq & \mu^{2}\left(t_{k}^{-}\right)\left[\tilde{z}\left(t_{k}^{-}\right)-D \int_{t_{k}-\delta}^{t_{k}} \tilde{z}(s) \mathrm{d} s\right]^{*} \\
& \times P_{1}\left[\tilde{z}\left(t_{k}\right)-D \int_{t_{k}-\delta}^{t_{k}} \tilde{z}(s) \mathrm{d} s\right] \\
= & V_{1}\left(t_{k}^{-}\right) .
\end{aligned}
$$

Hence, we can deduce that

$$
V\left(t_{k}\right) \leq V\left(t_{k}^{-}\right), \quad k \in \mathbb{Z}^{+} .
$$

From (46) and (49), we know that $V$ is monotonically nonincreasing for $t \in[T, \infty)$, which implies that

$$
V(t) \leq V(T), \quad t \geq T \text {. }
$$

From the definition of $V(t)$ in (32), we obtain that

$$
\mu^{2}(t) \lambda_{\min }\left(P_{2}\right)\|\tilde{z}(t)\|^{2} \leq V(t) \leq V_{0}<\infty, \quad t \geq 0,
$$

where $V_{0}=\max _{0 \leq s \leq T} V(s)$. It implies that

$$
\|\tilde{z}(t)\| \leq \frac{M}{\mu(t)}, \quad t \geq 0,
$$

where $M=\sqrt{V_{0} / \lambda_{\min }\left(P_{2}\right)}$. The proof is completed. 


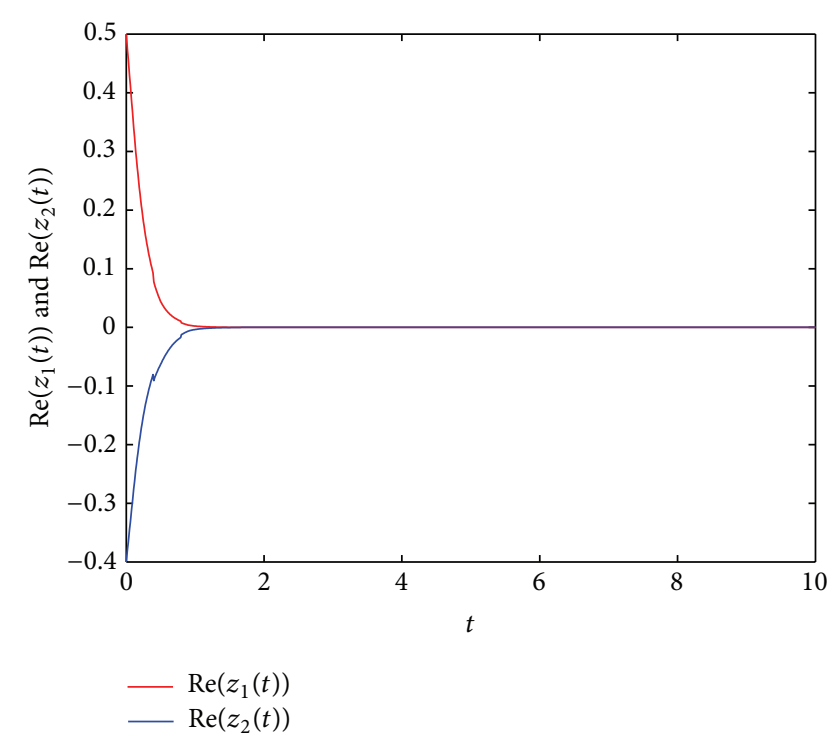

(a)

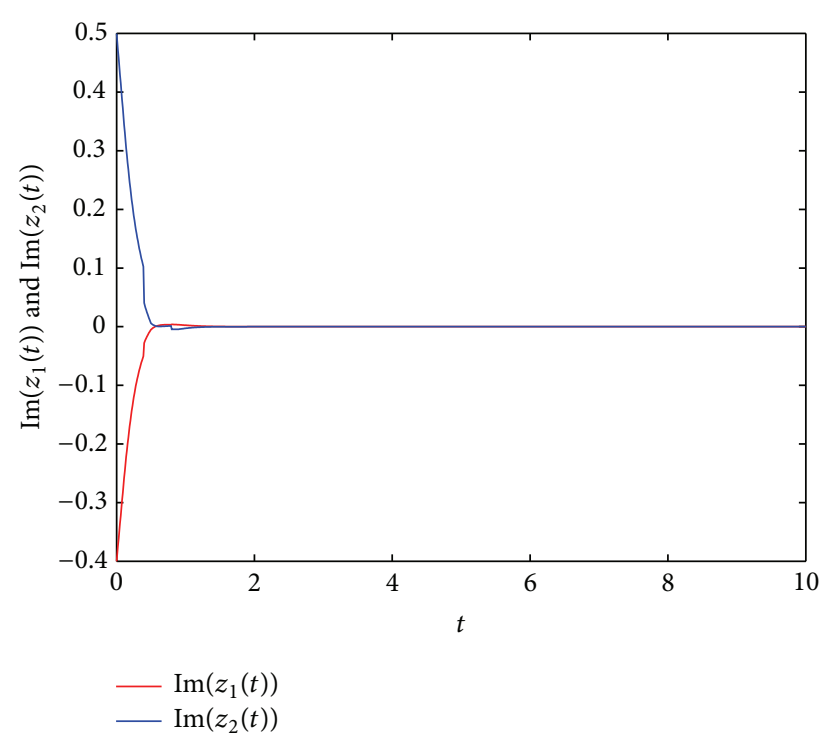

(b)

FIGURE 1: State trajectories of system (54).

Corollary 14. Under the conditions of Theorem 10, the equilibrium point of system (1) is globally exponentially stable, if there exist six positive definite Hermitian matrices $P_{1}, P_{2}, P_{3}$, $P_{4}, P_{5}$, and $P_{6}$, two real positive diagonal matrices $R_{1}$ and $R_{2}$, three complex matrices $Q_{1}, Q_{2}$, and $Q_{3}$, and a positive constant $\varepsilon$ such that conditions (29) and (30) in Theorem 13 are satisfied, where $\alpha=\varepsilon, \beta=e^{-\varepsilon \max \left\{\delta, \tau_{1}, \tau_{2}\right\}}$.

Proof. Let $\mu(t)=e^{\varepsilon t}(t \geq 0)$; then $\dot{\mu}(t) / \mu(t)=\varepsilon$, $\min \left\{\mu(t-\delta), \mu\left(t-\tau_{1}\right), \mu\left(t-\tau_{2}\right)\right\} / \mu(t)=\min \left\{e^{-\varepsilon \delta}, e^{-\varepsilon \tau_{1}}, e^{-\varepsilon \tau_{2}}\right\}=$ $e^{-\varepsilon \max \left\{\delta, \tau_{1}, \tau_{2}\right\}}$. Take $\alpha=\varepsilon, \beta=e^{-\varepsilon \max \left\{\delta, \tau_{1}, \tau_{2}\right\}}$, and $T=0$. Then it is obvious that condition (28) in Theorem 13 is satisfied. The proof is completed.

Corollary 15. Under the conditions of Theorem 10, the equilibrium point of system (1) is globally power-stable, if there exist six positive definite Hermitian matrices $P_{1}, P_{2}, P_{3}, P_{4}, P_{5}$, and $P_{6}$, two real positive diagonal matrices $R_{1}$ and $R_{2}$, three complex matrices $Q_{1}, Q_{2}$, and $Q_{3}$, and a positive constant $\varepsilon$ such that conditions (29) and (30) in Theorem 13 are satisfied, where $\alpha=\varepsilon / 2, \beta=2^{-\varepsilon}$.

Proof. Let $\mu(t)=t^{\varepsilon}$. For all $t \geq 2 \max \left\{1, \delta, \tau_{1}, \tau_{2}\right\}$, we have $\dot{\mu}(t) / \mu(t)=\varepsilon / t \leq \varepsilon / 2, \min \left\{\mu(t-\delta), \mu\left(t-\tau_{1}\right), \mu\left(t-\tau_{2}\right)\right\} / \mu(t)=$ $\left(1-\left(\max \left\{\delta, \tau_{1}, \tau_{2}\right\} / t\right)\right)^{\varepsilon} \geq 2^{-\varepsilon}$. Take $\alpha=\varepsilon / 2, \beta=2^{-\varepsilon}$, and $T=2 \max \left\{1, \delta, \tau_{1}, \tau_{2}\right\}$. Then it is obvious that condition (28) in Theorem 13 is satisfied. The proof is completed.

Corollary 16. Under the conditions of Theorem 10, the equilibrium point of system (1) is globally log-stable, if there exist six positive definite Hermitian matrices $P_{1}, P_{2}, P_{3}, P_{4}, P_{5}$, and $P_{6}$, two real positive diagonal matrices $R_{1}$ and $R_{2}$, three complex matrices $Q_{1}, Q_{2}$, and $Q_{3}$, and a positive constant $\varepsilon$ such that conditions (29) and (30) in Theorem 13 are satisfied, where $\alpha=\varepsilon / e, \beta=1 / \ln \left(e+\varepsilon \max \left\{\delta, \tau_{1}, \tau_{2}\right\}\right)$.
Proof. Let $\mu(t)=\ln (\varepsilon t+1)$. For all $t \geq((e-1) / \varepsilon)+$ $\max \left\{\delta, \tau_{1}, \tau_{2}\right\}$, we have $\dot{\mu}(t) / \mu(t)=\varepsilon /(\varepsilon t+1) \ln (\varepsilon t+1) \leq \varepsilon / e$, $\min \left\{\mu(t-\delta), \mu\left(t-\tau_{1}\right), \mu\left(t-\tau_{2}\right)\right\} / \mu(t)=\ln \left[\varepsilon\left(t-\max \left\{\delta, \tau_{1}, \tau_{2}\right\}\right)+\right.$ $1] / \ln (\varepsilon t+1) \geq 1 / \ln \left(e+\varepsilon \max \left\{\delta, \tau_{1}, \tau_{2}\right\}\right)$. Take $\alpha=\varepsilon / e, \beta=$ $1 / \ln \left(e+\varepsilon \max \left\{\delta, \tau_{1}, \tau_{2}\right\}\right)$, and $T=((e-1) / \varepsilon)+\max \left\{\delta, \tau_{1}, \tau_{2}\right\}$. Then it is obvious that condition (28) in Theorem 13 is satisfied. The proof is completed.

Remark 17. It is noted that LMIs (15), (29), and (30) are complex-valued, which cannot be directly handled via MATLAB LMI Toolbox. However, the authors in [33] give the result that a complex Hermitian matrix $L$ satisfies $L<0$ if and only if

$$
\left(\begin{array}{cc}
\operatorname{Re}(L) & \operatorname{Im}(L) \\
-\operatorname{Im}(L) & \operatorname{Re}(L)
\end{array}\right)<0 .
$$

Therefore, applying the result, the complex-valued LMIs (15), (29), and (30) can be turned into real-valued LMIs, which can be checked numerically using LMI toolbox in MATLAB.

\section{Numerical Examples}

The following two illustrative examples will demonstrate the effectiveness and superiority of our results.

Example 1. Consider the following two-neuron CVNNs with leakage, discrete, and distributed delays, described by

$$
\begin{aligned}
\dot{z}(t)= & -D z(t-\delta)+A f(z(t))+B f\left(z\left(t-\tau_{1}\right)\right) \\
& +C \int_{t-\tau_{2}}^{t} f(z(s)) \mathrm{d} s+h, \quad t \neq t_{k}, \quad t>0,
\end{aligned}
$$




$$
\begin{aligned}
\Delta z\left(t_{k}\right) & =z\left(t_{k}\right)-z\left(t_{k}^{-}\right) \\
& =E_{k}\left[z\left(t_{k}^{-}\right)-D \int_{t_{k}-\delta}^{t_{k}} z(s) \mathrm{d} s\right], \quad k \in \mathbb{Z}^{+},
\end{aligned}
$$

where $f_{1}(u)=f_{2}(u)=(1 / 20)(|u+1|-|u-1|), \delta=0.1$, $\tau_{1}=0.2, \tau_{2}=0.2, h=(0,0)^{T}, t_{k}=0.4 k, k \in \mathbb{Z}^{+}$, and the parameter matrices $D, A, B, C$, and $E_{k}$ are given as follows:

$$
\begin{aligned}
D & =\left(\begin{array}{ll}
3 & 0 \\
0 & 3
\end{array}\right), \quad A=\left(\begin{array}{cc}
0.5+i & -0.2-0.3 i \\
0.2+0.5 i & 1-0.5 i
\end{array}\right), \\
B & =\left(\begin{array}{cc}
0.4+0.3 i & -0.1-i \\
-1.2+i & -0.4-0.3 i
\end{array}\right), \\
C & =\left(\begin{array}{cc}
0.1-0.5 i & 0.6+0.4 i \\
0.2-0.5 i & 0.2
\end{array}\right), \\
E_{k} & =\left(\begin{array}{cc}
-0.3+0.2 i & 0 \\
0.1-0.6 i & -0.2+0.2 i
\end{array}\right), \quad k \in \mathbb{Z}^{+} .
\end{aligned}
$$

It can be verified that the activation functions $f_{1}$ and $f_{2}$ satisfy condition $(\mathrm{H} 1)$, and $\Gamma=\operatorname{diag}\{0.01,0.01\}$. Then LMI in Theorem 10 has the following feasible solution via the MATLAB LMI toolbox:

$$
\begin{aligned}
U_{1} & =\left(\begin{array}{cc}
19.0818+6.7535 i & -9.2220+2.2044 i \\
8.0728+0.4398 i & 19.0186+9.3571 i
\end{array}\right), \\
U_{2} & =\left(\begin{array}{cc}
4.7472-0.2821 i & -6.9954-6.3515 i \\
1.8858+0.8953 i & 4.5109+1.3211 i
\end{array}\right), \\
R & =\left(\begin{array}{cc}
161.0117 & 0 \\
0 & 129.7777
\end{array}\right) .
\end{aligned}
$$

Also there exists a constant $\varepsilon=0.1$, and by employing MATLAB LMI Toolbox, we can find the solutions to the LMIs in Corollary 14 as follows:

$$
\begin{aligned}
& P_{1}=\left(\begin{array}{cc}
12.4935 & -0.6208-3.5072 i \\
-0.6208+3.5072 i & 38.9645
\end{array}\right), \\
& P_{2}=\left(\begin{array}{cc}
16.5671 & -1.5742-1.2335 i \\
-1.5742+1.2335 i & 27.6891
\end{array}\right), \\
& P_{3}=\left(\begin{array}{cc}
43.4484 & -1.3614-5.3324 i \\
-1.3614+5.3324 i & 87.1860
\end{array}\right), \\
& P_{4}=\left(\begin{array}{cc}
12.8707 & -0.3721-2.5769 i \\
-0.3721+2.5769 i & 30.8832
\end{array}\right),
\end{aligned}
$$

$$
\begin{aligned}
P_{5} & =\left(\begin{array}{cc}
18.0542 & -0.3996+0.2524 i \\
-0.3996-0.2524 i & 16.1543
\end{array}\right), \\
P_{6} & =\left(\begin{array}{cc}
49.8004 & -0.2065+3.8010 i \\
-0.2065-3.8010 i & 48.9421
\end{array}\right) \\
Q_{1} & =\left(\begin{array}{cc}
-33.7168+0.0480 i & 3.0570-2.0568 i \\
2.2459+1.1090 i & -39.3508+0.0752 i
\end{array}\right) \\
Q_{2} & =\left(\begin{array}{cc}
-66.6664-1.5581 i & 6.8518-15.0791 i \\
1.2207+10.1224 i & -73.9430+1.7006 i
\end{array}\right) \\
Q_{3} & =\left(\begin{array}{cc}
-4.2664+0.1047 i & 0.4653-0.9119 i \\
0.3881+1.2719 i & 0.9145-0.0078 i
\end{array}\right) \\
R_{1} & =\left(\begin{array}{cc}
260.7956 & 0 \\
0 & 276.7577
\end{array}\right) \\
R_{2} & =\left(\begin{array}{cc}
293.9133 & 0 \\
0 & 256.1810
\end{array}\right)
\end{aligned}
$$

Hence, from Theorem 10 and Corollary 14, the unique equilibrium $(0,0)^{T}$ of system (54) is globally exponentially stable. Figure 1 depicts the real and imaginary parts of states of the considered system (54), where the initial condition is $z_{1}(t)=$ $0.5-0.4 i, z_{2}(t)=-0.4+0.5 i$.

Example 2. Consider the following two-neuron CVNNs with leakage, discrete, and distributed delays, described by

$$
\begin{aligned}
\dot{z}(t)= & -D z(t-\delta)+A f(z(t))+B f\left(z\left(t-\tau_{1}\right)\right) \\
& +C \int_{t-\tau_{2}}^{t} f(z(s)) \mathrm{d} s+h, \quad t \neq t_{k}, t>0, \\
\Delta z\left(t_{k}\right)= & z\left(t_{k}\right)-z\left(t_{k}^{-}\right) \\
= & E_{k}\left[z\left(t_{k}^{-}\right)-D \int_{t_{k}-\delta}^{t_{k}} z(s) \mathrm{d} s-\gamma\right], \quad k \in \mathbb{Z}^{+},
\end{aligned}
$$

where $f_{1}(u)=f_{2}(u)=(1 / 4)[\max \{0, \operatorname{Re}(u)\}+i \max \{0$, $\operatorname{Im}(u)\}], \delta=0.2, \tau_{1}=0.6, \tau_{2}=1, h=(1+i,-1+i)^{T}$, $\gamma=(0.0295+0.0623 i,-0.0604+0.0397 i)^{T}, t_{k}=0.8 k, k \in \mathbb{Z}^{+}$, and the parameter matrices $D, A, B, C$, and $E_{k}$ are given as follows:

$$
\begin{gathered}
D=\left(\begin{array}{ll}
4 & 0 \\
0 & 4
\end{array}\right), \quad A=\left(\begin{array}{cc}
-1+3 i & -1+2 i \\
-2-2 i & 1+0.5 i
\end{array}\right), \\
B=\left(\begin{array}{cc}
0.8+i & i \\
-1+0.5 i & 1+0.5 i
\end{array}\right), \quad C=\left(\begin{array}{cc}
1+i & 1-2 i \\
-1+i & 0.5+i
\end{array}\right), \\
E_{k}=\left(\begin{array}{cc}
-1 & 0.5 i \\
0.5 i & -0.5
\end{array}\right), \quad k \in \mathbb{Z}^{+} .
\end{gathered}
$$




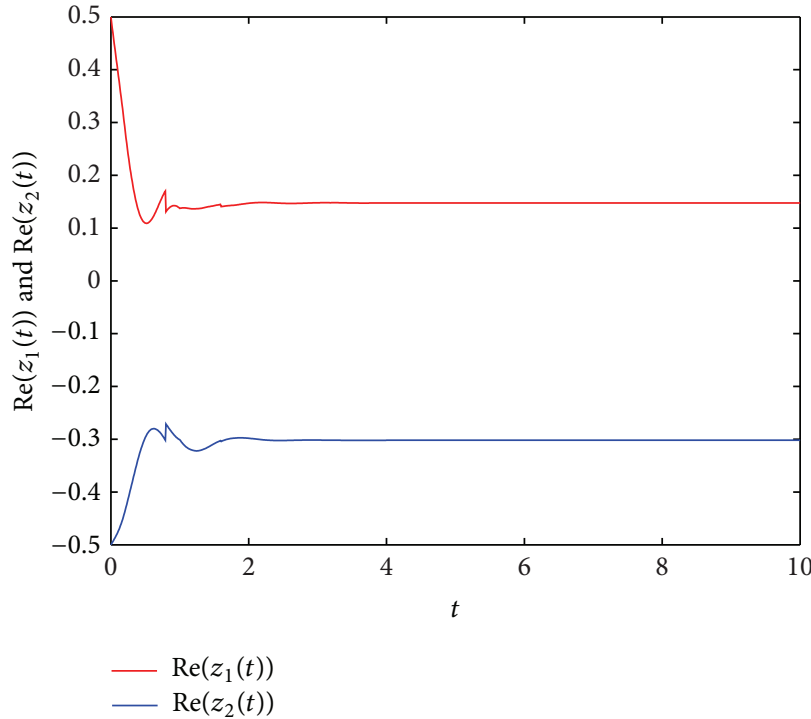

(a)

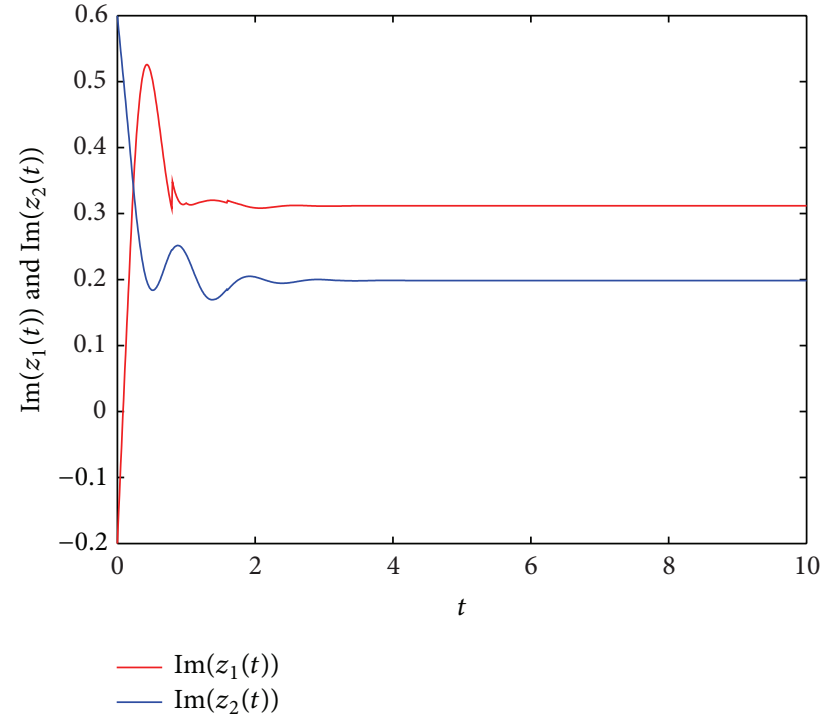

(b)

FiguRE 2: State trajectories of system (58) with $\delta=0.2$.

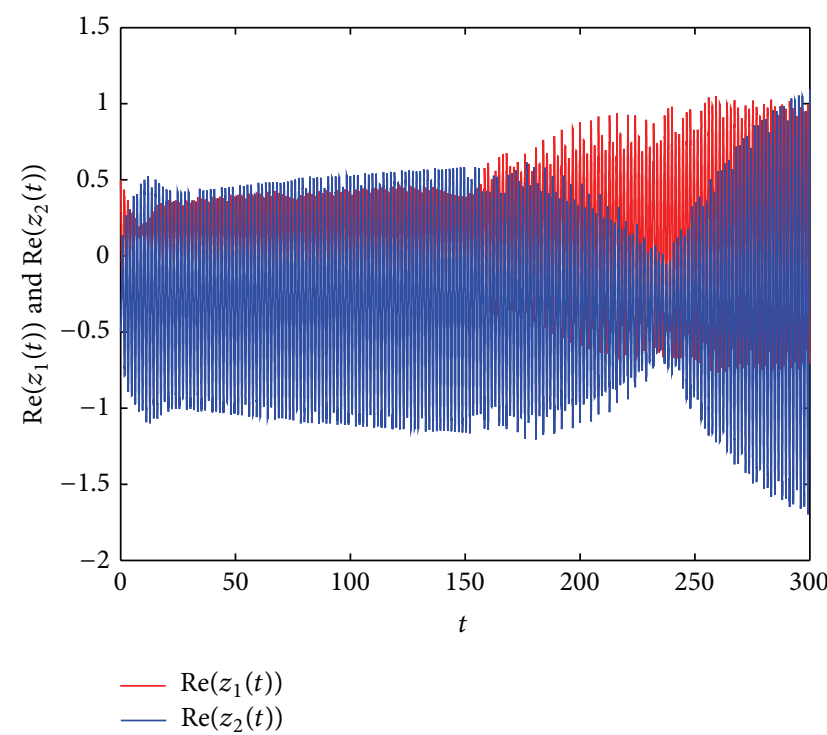

(a)

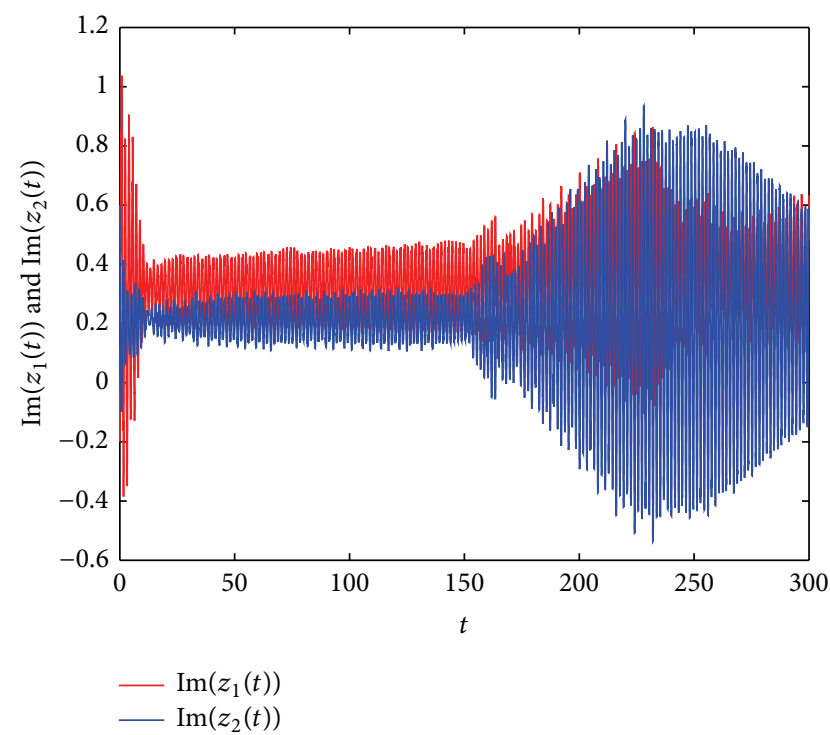

(b)

FIGURE 3: State trajectories of system (58) with $\delta=0.37$.

It can be verified that the activation functions $f_{1}$ and $f_{2}$ satisfy condition $(\mathrm{H} 1)$, and $\Gamma=\operatorname{diag}\{1 / 16,1 / 16\}$. Then LMI in Theorem 10 has the following feasible solution via the MATLAB LMI toolbox:

$$
\begin{aligned}
U_{1} & =\left(\begin{array}{cc}
2.9099-5.3413 i & 2.7078+7.2154 i \\
-2.5180+7.0990 i & 2.6469+0.8628 i
\end{array}\right), \\
U_{2} & =\left(\begin{array}{cc}
5.4524+4.8583 i & -11.7495+2.6580 i \\
-0.5273-7.2479 i & -0.8154-1.1665 i
\end{array}\right), \\
R & =\left(\begin{array}{cc}
79.4022 & 0 \\
0 & 37.6964
\end{array}\right)
\end{aligned}
$$

Also there exists a constant $\varepsilon=0.1$, and by employing MATLAB LMI Toolbox, we can find the solutions to LMIs in Corollary 15 as follows:

$$
\begin{aligned}
& P_{1}=\left(\begin{array}{cc}
1.6537 & 0.0741+0.0987 i \\
0.0741-0.0987 i & 2.5236
\end{array}\right), \\
& P_{2}=\left(\begin{array}{cc}
0.1984 & 0.0640-0.0118 i \\
0.0640+0.0118 i & 0.2985
\end{array}\right), \\
& P_{3}=\left(\begin{array}{cc}
21.5659 & 0.7498+1.4712 i \\
0.7498-1.4712 i & 32.7091
\end{array}\right),
\end{aligned}
$$




$$
\begin{aligned}
P_{4} & =\left(\begin{array}{cc}
0.6852 & 0.0304+0.0669 i \\
0.0304-0.0669 i & 1.1632
\end{array}\right), \\
P_{5} & =\left(\begin{array}{cc}
0.2419 & 0.0941-0.1415 i \\
0.0941+0.1415 i & 0.5347
\end{array}\right) \\
P_{6} & =\left(\begin{array}{cc}
7.7723 & -0.2071-0.7161 i \\
-0.2071+0.7161 i & 10.5317
\end{array}\right), \\
Q_{1} & =\left(\begin{array}{cc}
-0.7898+0.0341 i & -0.1269-0.5099 i \\
-0.1170+0.5513 i & -1.2707-0.0430 i
\end{array}\right), \\
Q_{2} & =\left(\begin{array}{cc}
-2.5965+0.0654 i & -0.5192-2.1874 i \\
-0.5396+2.4027 i & -4.2140-0.0583 i
\end{array}\right) \\
Q_{3} & =\left(\begin{array}{cc}
-0.0376+0.0030 i & -0.0029-0.0779 i \\
-0.0046+0.0854 i & -0.0596-0.0047 i
\end{array}\right) \\
R_{1} & =\left(\begin{array}{cc}
19.9880 & 0 \\
0 & 32.1768
\end{array}\right) \\
R_{2} & =\left(\begin{array}{cc}
6.8268 & 0 \\
0 & 14.7991
\end{array}\right) .
\end{aligned}
$$

Hence, from Theorem 10 and Corollary 15, system (58) has a unique equilibrium, which is globally exponentially powerstable. Figure 2 depicts the real and imaginary parts of states of the considered system (58), where the initial condition is $z_{1}(t)=0.5-0.2 i, z_{2}(t)=-0.5+0.6 i$.

Remark 18. If we take leakage delay $\delta \geq 0.37$ in system (58), one may check that LMIs in Corollary 15 do not have a feasible solution via MATLAB. In other words, our results cannot guarantee the stability of system (58) with $\delta \geq 0.37$. From the simulations, it is easy to check that the unique equilibrium point of system (58) with $\delta=0.37$ is not stable (see Figure 3 ); this implies that the effect of leakage delay on the dynamics of CVNNs cannot be ignored.

\section{Conclusion}

In this paper, the $\mu$-stability of impulsive CVNNs with leakage delay, discrete delay, and distributed delay has been investigated. Several sufficient conditions to ensure the existence, uniqueness, and global $\mu$-stability of the equilibrium point of the considered neural networks have been established in LMIs by applying the homeomorphism mapping principle of complex domain, constructing appropriate LyapunovKrasovskii functionals, and employing the free weighting matrix method. As direct applications of these results, several criteria on the exponential stability, power-stability and logstability have been obtained. Two numerical examples are given to illustrate the effectiveness of the proposed theoretical results.

\section{Conflict of Interests}

The authors declare that there is no conflict of interests regarding the publication of this paper.

\section{Acknowledgments}

This work was supported by the National Natural Science Foundation of China under Grants 61273021 and 11172247 and in part by the Natural Science Foundation Project of CQ cstc2013jjB40008.

\section{References}

[1] B. Shen, Z. Wang, D. Ding, and H. Shu, " $H_{\infty}$ state estimation for complex networks with uncertain inner coupling and incomplete measurements," IEEE Transactions on Neural Networks and Learning Systems, vol. 24, no. 12, pp. 2027-2037, 2013.

[2] S. Elmadssia, K. Saadaoui, and M. Benrejeb, "New delaydependent stability conditions for linear systems with delay," Systems Science and Control Engineering, vol. 1, no. 1, pp. 2-11, 2013.

[3] G. Wei, L. Wang, and F. Han, "A gain-scheduled approach to fault-tolerant control for discrete time stochastic delayed systems with randomly occurring actuator faults," Systems Science and Control Engineering, vol. 1, no. 1, pp. 82-90, 2013.

[4] J. Shen and J. Lam, "Decay rate constrained stability analysis for positive systems with discrete and distributed delays," Systems Science and Control Engineering, vol. 2, no. 1, pp. 7-12, 2014.

[5] M. Kermani and A. Sakly, "Stability analysis for a class of switched nonlinear time-delay systems," Systems Science and Control Engineering, vol. 2, no. 1, pp. 80-89, 2014.

[6] Q. Song, "Exponential stability of recurrent neural networks with both time-varying delays and general activation functions via LMI approach," Neurocomputing, vol. 71, no. 13-15, pp. 28232830, 2008.

[7] Y. Liu, Z. Wang, and X. Liu, "Asymptotic stability for neural networks with mixed time-delays: the discrete-time case," Neural Networks, vol. 22, no. 1, pp. 67-74, 2009.

[8] W. Weera and P. Niamsup, "Exponential stabilization of neutraltype neural networks with interval nondifferentiable and distributed time-varying delays," Abstract and Applied Analysis, vol. 2012, Article ID 101426, 21 pages, 2012.

[9] S. Arik, "A new condition for robust stability of uncertain neural networks with time delays," Neurocomputing, vol. 128, pp. 476482, 2014.

[10] K. Gopalsamy, "Leakage delays in BAM," Journal of Mathematical Analysis and Applications, vol. 325, no. 2, pp. 1117-1132, 2007.

[11] Y. Liu, Z. Wang, J. Liang, and X. Liu, "Synchronization of coupled neutral-type neural networks with jumping-modedependent discrete and unbounded distributed delays," IEEE Transactions on Cybernetics, vol. 43, no. 1, pp. 102-114, 2013.

[12] T. Lei, Q. Song, Z. Zhao, and J. Yang, "Synchronization of chaotic neural networks with leakage delay and mixed timevarying delays via sampled-data control," Abstract and Applied Analysis, vol. 2013, Article ID 290574, 10 pages, 2013.

[13] Q. Song and J. Cao, "Passivity of uncertain neural networks with both leakage delay and time-varying delay," Nonlinear Dynamics, vol. 67, no. 2, pp. 1695-1707, 2012.

[14] J. Liang, Z. Chen, and Q. Song, "State estimation for neural networks with leakage delay and time-varying delays," Abstract and Applied Analysis, vol. 2013, Article ID 289526, 9 pages, 2013.

[15] K. Gopalsamy, "Stability of artificial neural networks with impulses," Applied Mathematics and Computation, vol. 154, no. 3, pp. 783-813, 2004. 
[16] R. Rakkiyappan, P. Balasubramaniam, and J. Cao, "Global exponential stability results for neutral-type impulsive neural networks," Nonlinear Analysis, vol. 11, no. 1, pp. 122-130, 2010.

[17] S. Cai, P. Zhou, and Z. Liu, "Effects of time-varying impulses on the synchronization of delayed dynamical networks," Abstract and Applied Analysis, vol. 2013, Article ID 212753, 10 pages, 2013.

[18] Y. Fang, K. Yan, and K. Li, "Impulsive synchronization of a class of chaotic systems," Systems Science and Control Engineering, vol. 2, no. 1, pp. 55-60, 2014.

[19] X. Li, X. Fu, P. Balasubramaniam, and R. Rakkiyappan, "Existence, uniqueness and stability analysis of recurrent neural networks with time delay in the leakage term under impulsive perturbations," Nonlinear Analysis, vol. 11, no. 5, pp. 4092-4108, 2010.

[20] T. Chen and L. Wang, "Power-rate global stability of dynamical systems with unbounded time-varying delays," IEEE Transactions on Circuits and Systems II: Express Briefs, vol. 54, no. 8, pp. 705-709, 2007.

[21] T. Chen and L. Wang, "Global $\mu$-stability of delayed neural networks with unbounded time-varying delays," IEEE Transactions on Neural Networks, vol. 18, no. 6, pp. 1836-1840, 2007.

[22] X. Liu and T. Chen, "Robust $\mu$-stability for uncertain stochastic neural networks with unbounded time-varying delays," Physica A, vol. 387, no. 12, pp. 2952-2962, 2008.

[23] L. Yin and X. Fu, " $\mu$-stability of impulsive neural networks with unbounded time-varying delays and continuously distributed delays," Advances in Difference Equations, vol. 2011, Article ID 437842, 12 pages, 2011.

[24] L. Wang and T. Chen, "Multiple $\mu$-stability of neural networks with unbounded time-varying delays," Neural Networks, vol. 53, pp. 109-118, 2014.

[25] T. Nitta, "Orthogonality of decision boundaries in complexvalued neural networks," Neural Computation, vol. 16, no. 1, pp. 73-97, 2004.

[26] M. Faijul Amin and K. Murase, "Single-layered complexvalued neural network for real-valued classification problems," Neurocomputing, vol. 72, no. 4-6, pp. 945-955, 2009.

[27] A. Hirose, Complex-Valued Neural Networks: Theories and Applications, World Scientific, Singapore, 2004.

[28] V. S. H. Rao and G. R. Murthy, "Global dynamics of a class of complex valued neural networks," International Journal of Neural Systems, vol. 18, no. 2, pp. 165-171, 2008.

[29] W. Zhou and J. M. Zurada, "Discrete-time recurrent neural networks with complex-valued linear threshold neurons," IEEE Transactions on Circuits and Systems II: Express Briefs, vol. 56, no. 8, pp. 669-673, 2009.

[30] C. Duan and Q. Song, "Boundedness and stability for discretetime delayed neural network with complex-valued linear threshold neurons," Discrete Dynamics in Nature and Society, vol. 2010, Article ID 368379, 19 pages, 2010.

[31] X. Xu, J. Zhang, and J. Shi, "Exponential stability of complexvalued neural networks with mixed delays," Neurocomputing, vol. 128, pp. 483-490, 2014.

[32] M. Bohner, V. Sree Hari Rao, and S. Sanyal, "Global stability of complex-valued neural networks on time scales," Differential Equations and Dynamical Systems, vol. 19, no. 1-2, pp. 3-11, 2011.

[33] X. Chen and Q. Song, "Global stability of complex-valued neural networks with both leakage time delay and discrete time delay on time scales," Neurocomputing, vol. 121, pp. 254-264, 2013.
[34] X. Chen, Q. Song, X. Liu, and Z. Zhao, "Global $\mu$-stability of complex-valued neural networks with unbounded timevarying delays," Abstract and Applied Analysis, vol. 2014, Article ID 263847, 9 pages, 2014. 


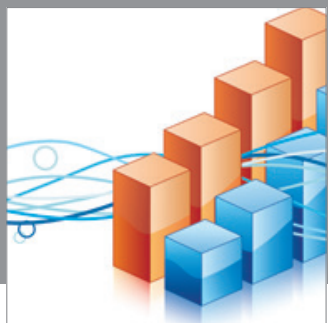

Advances in

Operations Research

mansans

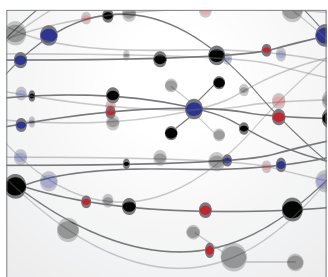

The Scientific World Journal
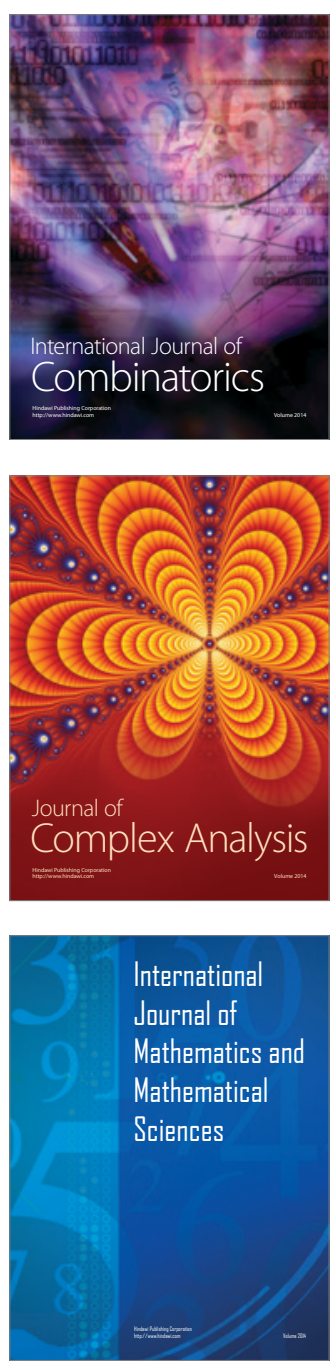
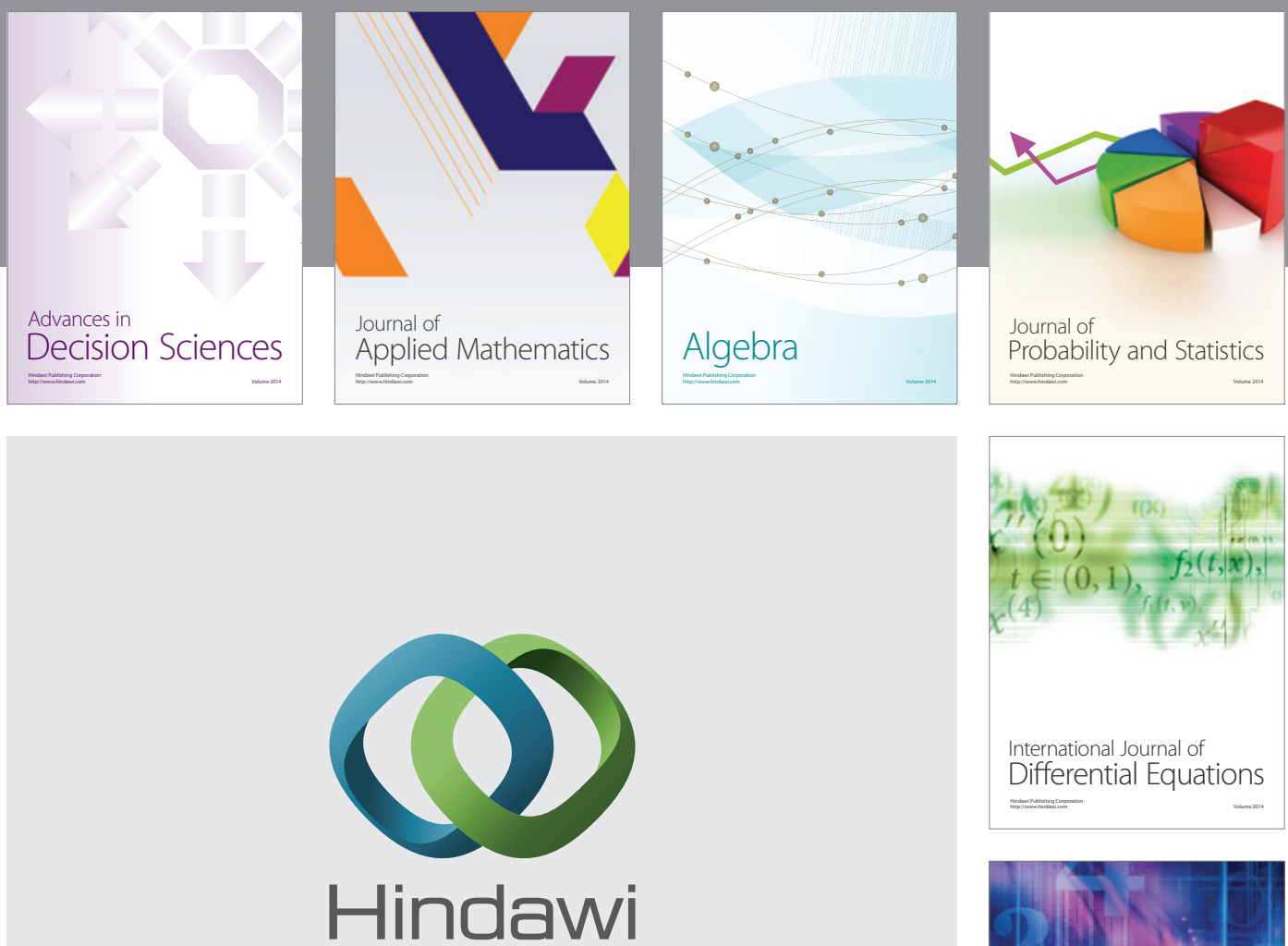

Submit your manuscripts at http://www.hindawi.com
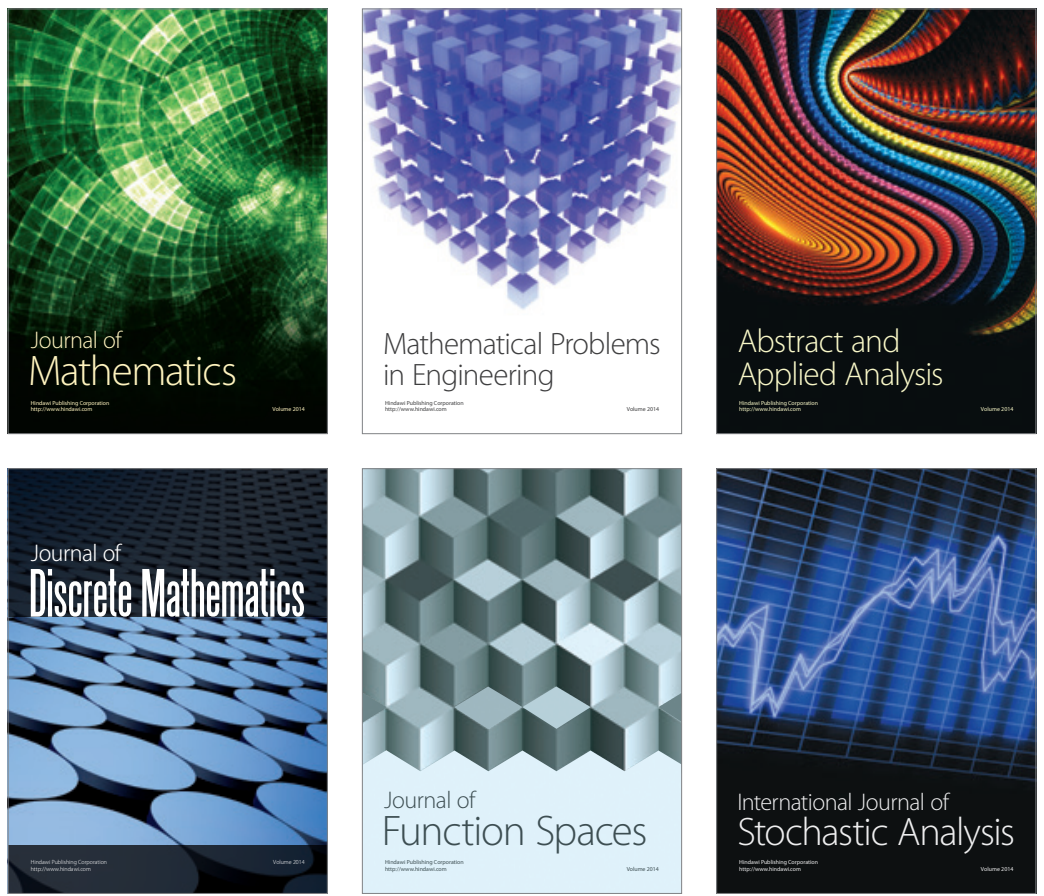

Journal of

Function Spaces

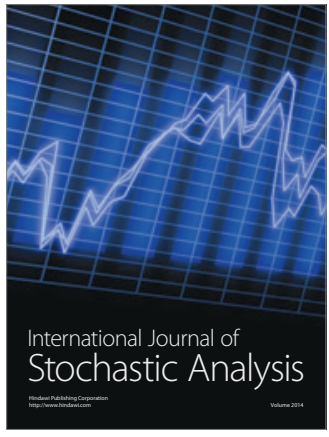

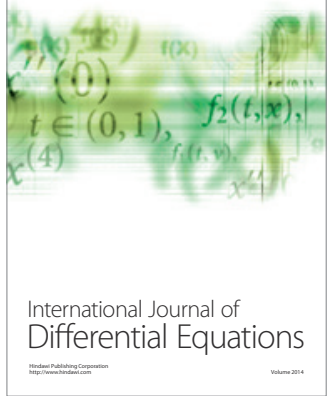
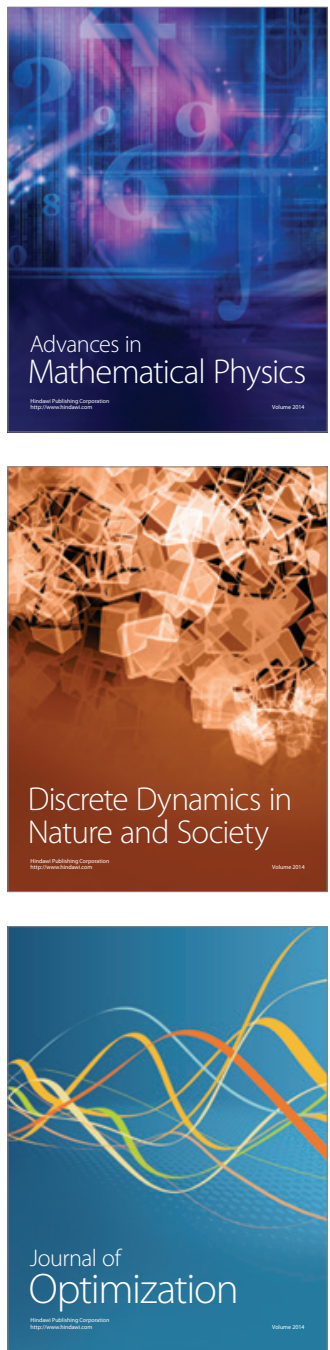